\title{
Length effects on interactive buckling in thin-walled rectangular hollow section struts
}

\author{
Jiajia Shen, M. Ahmer Wadee* \\ Department of Civil and Environmental Engineering, Imperial College London, \\ South Kensington Campus, London SW7 2AZ, UK
}

\begin{abstract}
A variational model formulated using analytical techniques describing the nonlinear coupling between local and global buckling modes within an elastic thin-walled rectangular hollow section strut is presented. A system of nonlinear differential and integral equations subject to boundary conditions is derived and solved using numerical continuation techniques. The nonlinear behaviour of four representative lengths is investigated, which are characterized by the post-buckling equilibrium paths. The numerical results from the variational model are validated using a nonlinear finite element model and largely show excellent comparisons, particularly for the practically important ultimate load and the initial post-buckling behaviour. Boundaries for the four distinct length-dependent zones are identified and the most unstable zone is demonstrated to have a considerably narrower length range than previously determined for practical corner boundary conditions within the cross-section.
\end{abstract}

Keywords: Coupled instabilities; Global buckling; Local buckling; Cellular buckling; Mode interaction; Variational modelling; Finite element validation

\section{Introduction}

The development of structural material and manufacturing technology alongside fundamentally better understanding of nonlinear mechanics have enabled structural forms to become increasingly slender. Buckling instability is practically always the governing failure mode of such structures [1, 2, 3, 4]. Nonlinear elastic stability theory [5, 6] has validated that slender columns and plates designed with linear theory are safe owing to their naturally stable post-buckling characteristics. According to linear theory, an equal stress triggering both local and global buckling would often seem to represent the most economical scheme. However, such approaches have been found to be inappropriate for thin-walled slender structures particularly when they become vulnerable to a variety

\footnotetext{
*Corresponding author

Email addresses: j.shen14@imperial.ac.uk (Jiajia Shen), a.wadee@imperial.ac.uk (M. Ahmer Wadee)
} 
of different nonlinear buckling phenomena 7, 8, 9, 10, 11, 12, 13, 14. Even though these modes may be stable when triggered in isolation, the interaction between individual modes can lead to unstable post-buckling behaviour and severe imperfection sensitivity 15, 16, 17, 18, 19, 20, 21, 22, 23, 24, 25, 26, 27, 28].

Early work on the interactive buckling of columns was conducted by van der Neut 8]. Using the theory originally devised by Koiter [5], van der Neut developed a relatively simplified model comprising two load-carrying flanges, which were simply-supported along their long edges, and a pair of rigid webs that only served to maintain the structural integrity but provided no longitudinal stiffness, as shown in Figure 1(a). Using this simpli-

(a)
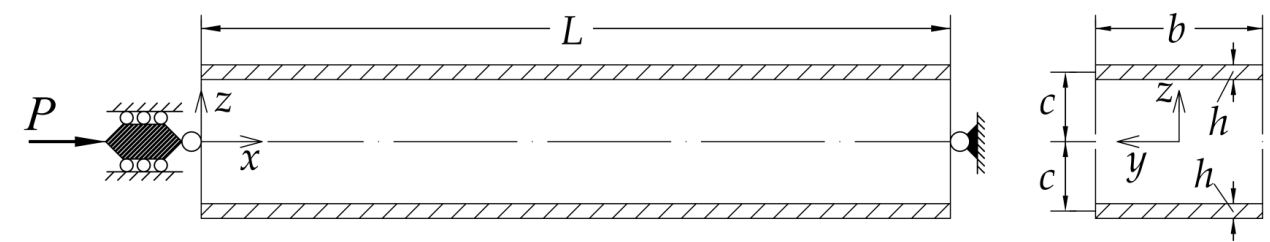

(b)

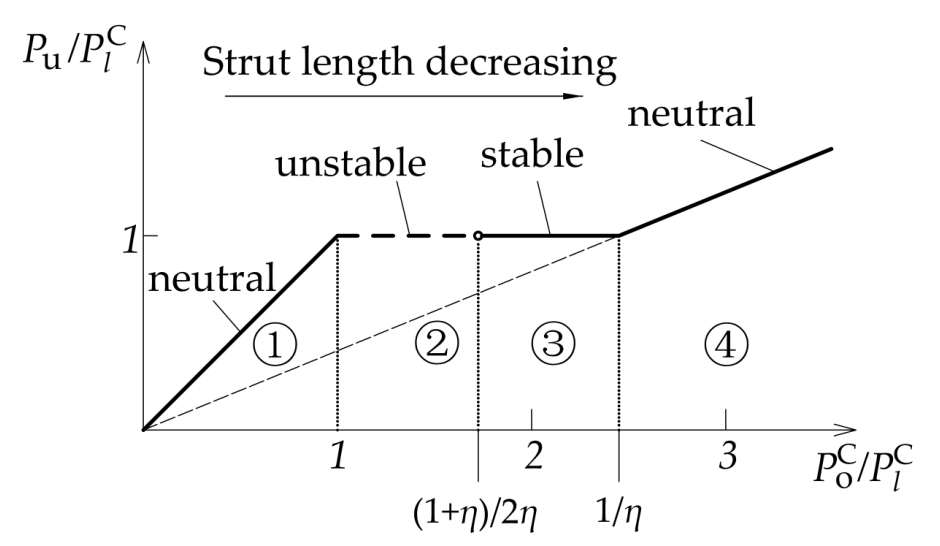

Figure 1: (a) The van der Neut model of an idealized thin-walled strut [8]. The strut comprises two load-carrying flanges with width $b$, thickness $h$, length $L$ with a pair of rigid webs of depth $2 c-h$ with no longitudinal stiffness; $P$ is the concentric axial load applied to the entire strut. (b) The van der Neut curve for the geometrically perfect case: $\eta$ is the stiffness reduction factor due to the local buckling of the flanges; $P_{\mathrm{u}}, P_{\mathrm{o}}^{\mathrm{C}}$ and $P_{1}^{\mathrm{C}}$ are the ultimate, the global buckling and the local buckling loads respectively.

fied model, the relationship between the properties of the initial post-buckling behaviour and the ratio of the global buckling load $P_{\mathrm{o}}^{\mathrm{C}}$ and the local buckling load $P_{1}^{\mathrm{C}}$, referred to currently as the 'van der Neut curve', was obtained, as shown in Figure 1(b).

Using largely the same approach, Koiter and Pignataro [29] investigated local-global mode interaction and developed an equivalent to the van der Neut curve for stiffened panels with one or multiple stringers. Compared with the idealized column, it was found that the unstable post-buckling range, labelled as zone 2 in Figure 1 (b), is considerably smaller in stiffened panels. Owing to the limitations of the asymptotic method used, only very 
restricted information about the nonlinear behaviour was presented apart from the initial post-buckling response.

Byskov [30] applied the asymptotic expansion method developed previously in [20] to van der Neut's column. However, owing to the limitation of the method [31, 32], which is only valid when the post-buckling behaviour of the local mode is neutral or unstable, the errors were relatively large and the results were only meaningful in close proximity of the boundary between zones 1 and 2 .

Sridharan and his collaborators [33, 34, 35, 36. used the finite strip method 37, 38. in conjunction with Koiter's theory to analyse mode interaction in thin-walled structural members with different cross-sections. As for the global mode, both the purely flexural and the flexural-torsional buckling modes were considered. The numerical results showed good comparisons with existing experimental results and substantial erosion in load-carrying capacity due to imperfections was identified. Using a similar approach, Møllmann and Goltermann [39, 40] investigated the interactive buckling behaviour of an I-section beam under pure bending and a box-section column under pure compression. Substantial reductions of up to $50 \%$ in the load-carrying capacity were observed in both examples due to the mode interaction.

The interactive buckling of square hollow section columns using the commercial finite element (FE) package ABAQUs was investigated in [41]. Three example columns with typical slendernesses were analysed, where (i) global buckling and (ii) local buckling were clearly critical, and (iii) where global and local buckling were triggered simultaneously. Unstable post-buckling paths due to the mode interaction were observed in all the example columns. The localization of the post-buckling mode due to the interaction was also highlighted. Since that work principally focused on the effects of initial imperfections, residual stresses and material plasticity on the ultimate load and post-buckling behaviour, no further details about the evolution of the modal interaction, equilibrium paths, slenderness effects nor the underlying mechanism were presented.

More recently, Wadee and his collaborators have developed a series of variational models using analytical techniques to investigate mode interaction in I-section struts [13, 42] and stiffened plates [14]. These variational models exhibit very good comparisons with FE models using shell elements and existing experimental results [11]. Using the numerical continuation method, the evolution of the modal interaction in the post-buckling range has been captured well by these models. In particular, for some geometric ranges, snap-backs in the response, showing sequential destabilization and restabilization and a progressive spreading of the local buckling mode from the mid-span, known as cellular buckling [43], have been predicted, which have also been observed in physical experiments on thin-walled struts 11] and beams [12]. Using the validated variational models, the effects of the global and local slenderness on the mode interaction behaviour have been investigated. Equivalent van der Neut-type curves for I-section struts [27, 44] and stiffened plates [26], with more detailed information about the post-buckling behaviour has been presented, thus identifying the most unstable parametric spaces of the geometries.

Mode interaction has also been observed in physical experiments on hollow section columns [45, 46, 47, 48, 49, 50]. However, these particular investigations have principally 
focused on the ultimate load capacity and the behaviour in the inelastic range. Therefore, limited information on the underlying mechanisms of mode interaction has been presented. However, in the physical tests on I-section struts under pure compression [51, 11] and Isection beams under uniform bending [12], more details about the evolution of the interactive buckling, in terms of the amplitude modulation and the change in the wavelength of the local buckling profile with the increase of the applied loading deformation, were highlighted.

In the authors' recent work [52], a variational model describing mode interaction in long rectangular hollow section struts was developed and showed excellent comparisons with FE models developed within the commercial package ABAQus [53]. The work identified that when the local buckling load is close to the global buckling load, such a system can exhibit a violent destabilization once the peak load is reached. The present work develops the model to include the scenarios where local buckling is critical to investigate how shorter strut lengths affect the nonlinear modal coupling.

The interactive buckling responses of four representative struts that are currently defined qualitatively in sequence as the length is progressively reduced as 'long', 'transitional', 'intermediate' and 'short' length struts, thus corresponding to the four different zones shown in Figure 1(b) respectively, are studied. The characteristic interactive buckling paths are determined and a progressive change in the local buckling wavelength is found. The numerical results from the variational model largely show excellent comparisons with numerical results obtained using a nonlinear FE model developed within ABAQUS in terms of the ultimate load and the initial post-buckling behaviour. A parametric study on the strut length identifies the boundaries of the four distinct length-dependent domains and places the current results within the context of the classical work by van der Neut. The current work facilitates a better understanding of the behaviour of thin-walled rectangular hollow section struts that exhibit local-global mode interaction with different slendernesses and in future will allow for the establishment of guidance for structural engineers designing such elements that are vulnerable to such phenomena.

\section{Development of the variational model}

A thin-walled rectangular hollow section strut of length $L$ with simply-supported boundary conditions being loaded by a concentric force $P$ is considered, as shown in Figure 2, The web depth and thickness are $d$ and $t_{\mathrm{w}}$ respectively; the flange width and thickness are $b$ and $t_{\mathrm{f}}$ respectively. The material properties of the strut are assumed to be linearly elastic, homogeneous and isotropic with Young's modulus $E$, Poisson's ratio $\nu$ and shear modulus $G=E /[2(1+\nu)]$. The joints between the webs and the flanges are assumed to be rigid, hence the following two assumptions are satisfied [54]: (i) the webs and the flanges at their common edges remain orthogonal during buckling; (ii) the wavelengths of the local buckling mode, which simultaneously appear in all constituent plates, are the same. 
(a) Simply-supported strut under pure compression

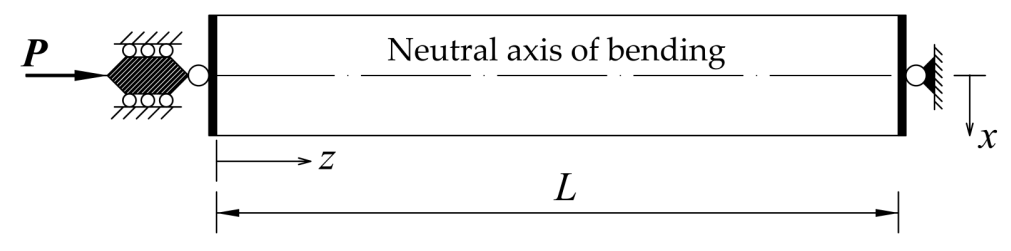

(b) Cross-section

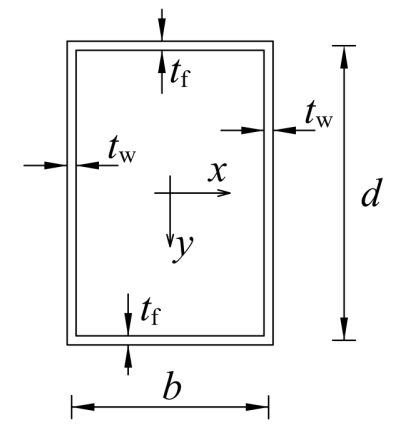

Figure 2: (a) Plan view of the rectangular hollow section strut of length $L$ under the concentric axial load $P$. The lateral and longitudinal coordinates are $x$ and $z$ respectively. (b) Cross-section geometry of the strut; the vertical axis coordinate being $y$.

\subsection{Modal descriptions}

The formulation begins with the description of both the global and the local modal displacements based on a recent purely numerical study [55]. Since previous studies [56, 57, 13, 58, 59] have shown that it is essential to include the components of bending shear strain in the total potential energy formulation to model the interactive buckling behaviour, Timoshenko beam theory is adopted. The global mode is decomposed into two components, a pure lateral displacement $W$ and a pure rotation of the plane sections $\theta$, see Figure [3(a), known as the 'sway' and 'tilt' modes [60, 56] respectively. The lateral displacement $W$ and the corresponding rotation $\theta$ are defined by the following expressions:

$$
W(z)=-q_{s} L \sin \left(\frac{\pi z}{L}\right), \quad \theta(z)=-q_{t} \pi \cos \left(\frac{\pi z}{L}\right),
$$

where $q_{s}$ and $q_{t}$ are the generalized coordinates for the sway and tilt modes respectively. The shear strain in the flanges is given by the following expression:

$$
\gamma_{x z}=\frac{\mathrm{d} W}{\mathrm{~d} z}-\theta=-\left(q_{s}-q_{t}\right) \pi \cos \left(\frac{\pi z}{L}\right) .
$$

The local buckling mode, including out-of-plane and in-plane displacement components, shown in Figure 3(b), is expressed as a combination of the local modes in the pure local buckling case and the case where global buckling is critical:

$$
\begin{aligned}
w_{\mathrm{f}}(x, z) & =f_{1 \mathrm{f}}(x) w_{1}(z)+f_{2 \mathrm{f}}(x) w_{2}(z), \\
w_{\mathrm{wc}}(y, z) & =f_{1 \mathrm{wc}}(y) w_{1}(z)+f_{2 \mathrm{wc}}(y) w_{2}(z), \\
w_{\mathrm{wt}}(y, z) & =f_{1 \mathrm{wt}}(y) w_{1}(z)+f_{2 \mathrm{wt}}(y) w_{2}(z), \\
u_{\mathrm{f}}(x, z) & =g_{1 \mathrm{f}}(x) u_{1}(z)+g_{2 \mathrm{f}}(x) u_{2}(z), \\
u_{\mathrm{wc}}(y, z) & =g_{1 \mathrm{wc}}(y) u_{1}(z)+g_{2 \mathrm{wc}}(y) u_{2}(z), \\
u_{\mathrm{wt}}(y, z) & =g_{1 \mathrm{wt}}(y) u_{1}(z)+g_{2 \mathrm{wt}}(y) u_{2}(z),
\end{aligned}
$$


(a)

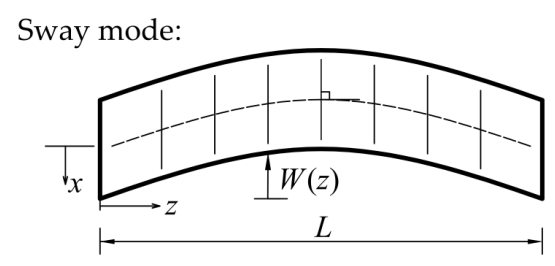

Tilt mode:

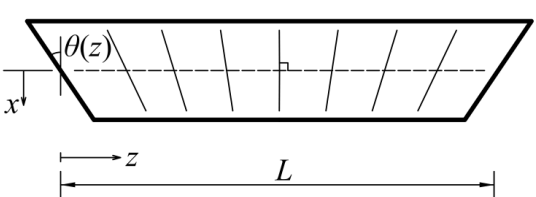

(b) Out-of-plane:
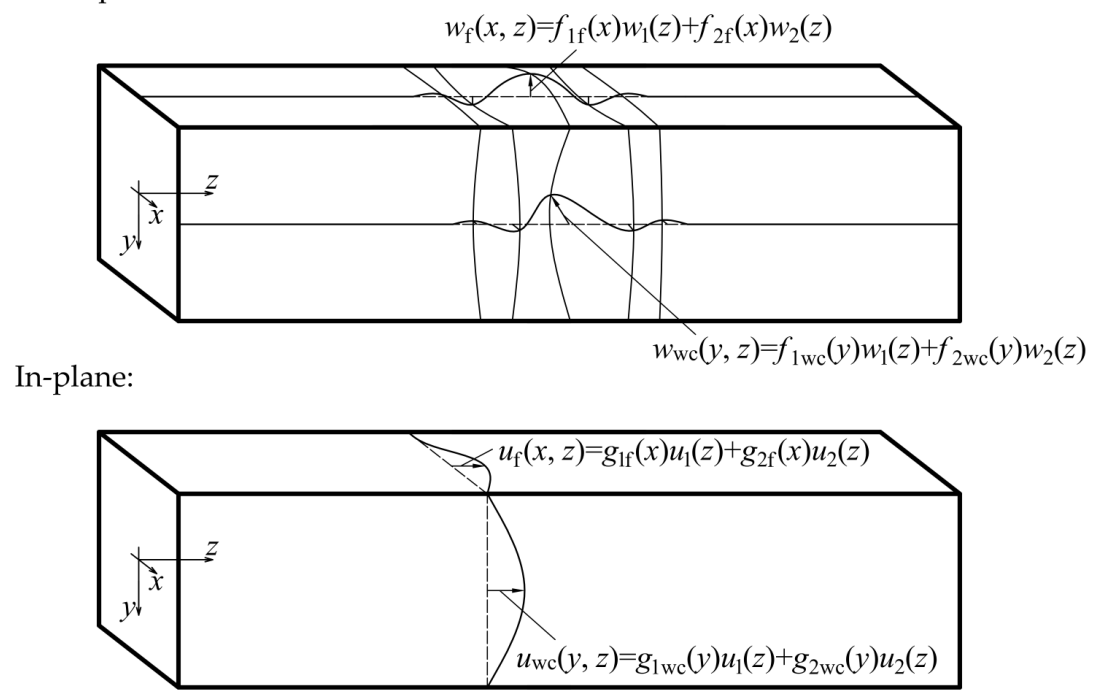

Figure 3: (a) Sway and tilt components of the global buckling mode bending about the weak axis $y$. (b) Out-of-plane local mode in the flanges $w_{\mathrm{f}}(x, z)$ and in the more compressed web $w_{\mathrm{wc}}(y, z)$. Also shown are the in-plane local mode in the flanges $u_{\mathrm{f}}(x, z)$ and in the more compressed web $u_{\mathrm{wc}}(y, z)$. 
where $f$ and $g$ are the cross-section components for the out-of-plane and in-plane components respectively; $w_{i}$ and $u_{i}$, where $i=\{1,2\}$, are the longitudinal out-of-plane and inplane displacement components respectively. Subscripts 1 and 2 represent the cases where local and global buckling are critical respectively, as shown in Figure 4 . The subscripts

(a) Local mode 1

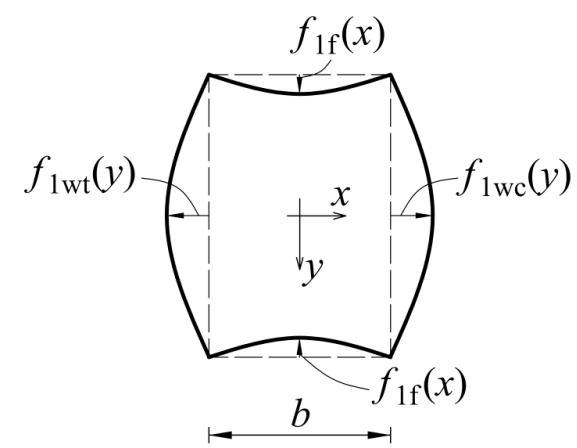

(b) Local mode 2



Figure 4: Local out-of-plane displacement fields of the cross-section. (a) Pure local buckling case. (b) Interactive buckling case where global buckling is critical.

' $\mathrm{f}$ ' and ' $\mathrm{w}$ ' represent the flanges and webs respectively; subscripts ' $c$ ' and ' $\mathrm{t}$ ' represent the more and less compressed webs respectively.

From a recent numerical study [55], it was determined that the cross-section shape functions for the out-of-plane and in-plane components, $f$ and $g$, are approximately the same. Therefore, these components are in fact assumed to be the same currently, i.e. $g_{i \mathrm{f}}=f_{i \mathrm{f}}, g_{i \mathrm{wc}}=f_{i \mathrm{wc}}$ and $g_{i \mathrm{wt}}=f_{i \mathrm{wt}}$. The cross-section out-of-plane components $f$, as shown in Figure 4, are estimated by applying kinematic and static boundary conditions for each plate. For the pure local critical mode, as shown in Figure 4(a), it is assumed that the out-of-plane displacement in the webs $f_{1 \mathrm{w}}$ has the functional form that is derived from the conditions of a simply-supported strut (a cosine wave with the current coordinate system) and a beam under pure bending (a parabola from applying Euler-Bernoulli beam theory), thus:

$$
f_{1 \mathrm{wc}}=f_{1 \mathrm{wt}}=A_{10} \cos \frac{\pi y}{d}+\left(1-A_{10}\right)\left(1-\frac{4 y^{2}}{d^{2}}\right) .
$$

For the out-of-plane displacement in the flanges $f_{1 \mathrm{f}}$, the functional form is derived from a simply-supported beam with equal and opposite end moments arising from the transfer of moment at the flange--web joints. This naturally leads to the following parabolic form:

$$
f_{1 \mathrm{f}}=B_{10}\left(\frac{x^{2}}{b^{2}}-\frac{1}{4}\right)
$$

The coefficients $A_{10}$ in $f_{1 \mathrm{wc}}$ and $B_{10}$ in $f_{1 \mathrm{f}}$ are determined by applying appropriate boundary conditions at the junctions. The form of $f_{1 \mathrm{wc}}$ and $f_{1 \mathrm{f}}$ automatically satisfies the natural boundary conditions at the junctions, i.e. $f_{1 \mathrm{wc}}( \pm d / 2)=0$ and $f_{1 \mathrm{f}}( \pm b / 2)=0$. 
Owing to the symmetric form of the functions, the junction between the flange and the web with the coordinate $(x=b / 2, y=d / 2)$ is considered. For rotational continuity, the following boundary condition also needs to be satisfied:

$$
\theta_{1 \mathrm{f}}=\left.\frac{\mathrm{d} f_{1 \mathrm{f}}}{\mathrm{d} x}\right|_{x=b / 2}=\theta_{1 \mathrm{wc}}=\left.\frac{\mathrm{d} f_{1 \mathrm{wc}}}{\mathrm{d} y}\right|_{y=d / 2} .
$$

Moreover, moment equilibrium at the junction leads to a further condition:

$$
M_{1 \mathrm{f}}(x=b / 2)+M_{1 \mathrm{wc}}(y=d / 2)=0,
$$

where:

$$
\begin{gathered}
M_{1 \mathrm{f}}(x=b / 2)=\left[D_{\mathrm{f}}\left(\frac{\partial^{2} w_{1 \mathrm{f}}}{\partial x^{2}}+\nu \frac{\partial^{2} w_{1 \mathrm{f}}}{\partial z^{2}}\right)\right]_{x=b / 2}=\left.D_{\mathrm{f}} w_{1} \frac{\mathrm{d}^{2} f_{1 \mathrm{f}}}{\mathrm{d} x^{2}}\right|_{x=b / 2}, \\
M_{1 \mathrm{wc}}(y=d / 2)=\left[D_{\mathrm{w}}\left(\frac{\partial^{2} w_{1 \mathrm{wc}}}{\partial y^{2}}+\nu \frac{\partial^{2} w_{1 \mathrm{wc}}}{\partial z^{2}}\right)\right]_{y=d / 2}=\left.D_{\mathrm{w}} w_{1} \frac{\mathrm{d}^{2} f_{1 \mathrm{wc}}}{\mathrm{d} y^{2}}\right|_{y=d / 2},
\end{gathered}
$$

where $D_{\mathrm{f}}=E t_{\mathrm{f}}^{3} /\left[12\left(1-\nu^{2}\right)\right]$ and $D_{\mathrm{w}}=E t_{\mathrm{w}}^{3} /\left[12\left(1-\nu^{2}\right)\right]$ are the flexural rigidities of the individual flange and web plates respectively. The terms related to the Poisson's ratio $\nu$ disappear due to the fact that $f_{1 \mathrm{f}}=f_{1 \mathrm{wc}}=0$ at the junction. Based on the boundary conditions presented in Eqs. (6) and (17), the coefficients $A_{10}$ and $B_{10}$ are thus:

$$
A_{10}=\frac{4\left(\phi_{\mathrm{c}} \phi_{\mathrm{t}}^{3}+1\right)}{4+\phi_{\mathrm{c}} \phi_{\mathrm{t}}^{3}(1-\pi)}, \quad B_{10}=\frac{4 \pi}{\phi_{\mathrm{c}}\left[4+\phi_{\mathrm{c}} \phi_{\mathrm{t}}^{3}(1-\pi)\right]},
$$

where the cross-section aspect ratio $\phi_{\mathrm{c}}=d / b$, and the flange-web thickness ratio $\phi_{\mathrm{t}}=t_{\mathrm{f}} / t_{\mathrm{w}}$. It should be stressed that the above assumption for $f_{1 \mathrm{f}}$ and $f_{1 \mathrm{w}}$ is only valid for rectangular sections $(d>b)$, where the critical stress in the webs is smaller than in the flanges.

The cross-section components describing mode interaction where global buckling is critical, as shown in Figure $4(\mathrm{~b})$, can also be determined in a similar way - the derivation can be found in [52] and the components are thus:

$$
\begin{aligned}
f_{2 \mathrm{wc}} & =\frac{2\left(2 \phi_{\mathrm{c}} \phi_{\mathrm{t}}^{3}+1\right)}{2+\phi_{\mathrm{c}} \phi_{\mathrm{t}}^{3}(4-\pi)} \cos \frac{\pi y}{d}-\frac{\pi \phi_{\mathrm{c}} \phi_{\mathrm{t}}^{3}}{2+\phi_{\mathrm{c}} \phi_{\mathrm{t}}^{3}(4-\pi)}\left(1-\frac{4 y^{2}}{d^{2}}\right) \\
f_{2 \mathrm{f}} & =\frac{-2 \pi}{\phi_{\mathrm{c}}\left[2+\phi_{\mathrm{c}} \phi_{\mathrm{t}}^{3}(4-\pi)\right]}\left(\frac{x}{b}+\frac{1}{2}\right)^{2}+\frac{2 \pi}{\phi_{\mathrm{c}}\left[2+\phi_{\mathrm{c}} \phi_{\mathrm{t}}^{3}(4-\pi)\right]}\left(\frac{x}{b}+\frac{1}{2}\right)^{3}, \\
f_{2 \mathrm{wt}} & =0 .
\end{aligned}
$$

\subsection{Potential energy formulation}

The total potential energy $V$ comprises the contributions of the bending strain energy from the global buckling mode and the local out-of-plane displacement of individual plates due to the local buckling mode, the membrane strain energy arising from axial and shear strains, and the work done by the external load $P \mathcal{E}$, where $\mathcal{E}$ is the total end-shortening. The formulation of the total potential energy functional $V$ follows a similar approach as found in [52], but currently accounts for the scenarios where either local or global buckling may be critical. 


\subsubsection{Strain energy due to bending}

It should be noted that only bending strain energy stored in the webs contributes to the global bending energy $U_{\mathrm{b}, \mathrm{o}}$ from the sway mode, since the membrane energy stored in the flanges accounts for the effect of global bending from the tilt mode, see Figure [3(a). Therefore, the global bending energy can be expressed thus:

$$
U_{\mathrm{b}, \mathrm{o}}=2 \int_{0}^{L} \frac{E I_{\mathrm{w}}}{2} \ddot{W}^{2} \mathrm{~d} z=E I_{\mathrm{w}} \int_{0}^{L} q_{s}^{2} \frac{\pi^{4}}{L^{2}} \sin ^{2} \frac{\pi z}{L} \mathrm{~d} z
$$

where $I_{\mathrm{w}}=d t_{\mathrm{w}}^{3} / 12$, which is the second moment of area about the local weak neutral axis of the web and dots represent differentiation with respect to $z$. The factor of 2 is included to account for both webs.

The local bending energy stored in both flanges, the more compressed web and the less compressed web can be determined by the standard expressions [61]:

$$
\begin{gathered}
U_{\mathrm{b}, \mathrm{f}}=D_{\mathrm{f}} \int_{0}^{L} \int_{-b / 2}^{b / 2}\left\{\left(\frac{\partial^{2} w_{\mathrm{f}}}{\partial z^{2}}+\frac{\partial^{2} w_{\mathrm{f}}}{\partial x^{2}}\right)^{2}\right. \\
\left.-2(1-\nu)\left[\frac{\partial^{2} w_{\mathrm{f}}}{\partial z^{2}} \frac{\partial^{2} w_{\mathrm{f}}}{\partial x^{2}}-\left(\frac{\partial^{2} w_{\mathrm{f}}}{\partial z \partial x}\right)^{2}\right]\right\} \mathrm{d} x \mathrm{~d} z, \\
U_{\mathrm{b}, \mathrm{wc}}=\frac{D_{\mathrm{w}}}{2} \int_{0}^{L} \int_{-d / 2}^{d / 2}\left\{\left(\frac{\partial^{2} w_{\mathrm{wc}}}{\partial z^{2}}+\frac{\partial^{2} w_{\mathrm{wc}}}{\partial y^{2}}\right)^{2}\right. \\
\left.-2(1-\nu)\left[\frac{\partial^{2} w_{\mathrm{wc}}}{\partial z^{2}} \frac{\partial^{2} w_{\mathrm{wc}}}{\partial y^{2}}-\left(\frac{\partial^{2} w_{\mathrm{wc}}}{\partial z \partial y}\right)^{2}\right]\right\} \mathrm{d} y \mathrm{~d} z, \\
U_{\mathrm{b}, \mathrm{wt}}=\frac{D_{\mathrm{w}}}{2} \int_{0}^{L} \int_{-d / 2}^{d / 2}\left\{\left(\frac{\partial^{2} w_{\mathrm{wt}}}{\partial z^{2}}+\frac{\partial^{2} w_{\mathrm{wt}}}{\partial y^{2}}\right)^{2}\right. \\
\left.-2(1-\nu)\left[\frac{\partial^{2} w_{\mathrm{wt}}}{\partial z^{2}} \frac{\partial^{2} w_{\mathrm{wt}}}{\partial y^{2}}-\left(\frac{\partial^{2} w_{\mathrm{wt}}}{\partial z \partial y}\right)^{2}\right]\right\} \mathrm{d} y \mathrm{~d} z .
\end{gathered}
$$

By substituting $w_{\mathrm{f}}$ from Eq. (3) into Eq. (15), the explicit expression for the local bending energy in the flanges is written thus:

$$
\begin{aligned}
U_{\mathrm{b}, \mathrm{f}}=D_{\mathrm{f}} \int_{0}^{L}[ & \left\{f_{1 \mathrm{f}}^{2}\right\}_{x} \ddot{w}_{1}^{2}+\left\{f_{2 \mathrm{f}}^{2}\right\}_{x} \ddot{w}_{2}^{2}+\left\{f_{1 \mathrm{f}}^{\prime \prime 2}\right\}_{x} w_{1}^{2}+\left\{f_{2 \mathrm{f}}^{\prime \prime 2}\right\}_{x} w_{2}^{2} \\
& +2\left\{f_{1 \mathrm{f}} f_{2 \mathrm{f}}\right\}_{x} \ddot{w}_{1} \ddot{w}_{2}+2\left\{f_{1 \mathrm{f}}^{\prime \prime} f_{2 \mathrm{f}}^{\prime \prime}\right\}_{x} w_{1} w_{2}+2 \nu\left\{f_{1 \mathrm{f}} f_{1 \mathrm{f}}^{\prime \prime}\right\}_{x} \ddot{w}_{1} w_{1} \\
& +2 \nu\left\{f_{2 \mathrm{f}} f_{2 \mathrm{f}}^{\prime \prime}\right\}_{x} \ddot{w}_{2} w_{2}+2 \nu\left\{f_{1 \mathrm{f}} f_{2 \mathrm{f}}^{\prime \prime}\right\}_{x} \ddot{w}_{1} w_{2}+2 \nu\left\{f_{1 \mathrm{f}}^{\prime \prime} f_{2 \mathrm{f}}\right\}_{x} w_{1} \ddot{w}_{2} \\
& \left.+2(1-\nu)\left(\left\{f_{1 \mathrm{f}}^{\prime 2}\right\}_{x} \dot{w}_{1}^{2}+\left\{f_{2 \mathrm{f}}^{\prime 2}\right\}_{x} \dot{w}_{2}^{2}+2\left\{f_{1 \mathrm{f}}^{\prime} f_{2 \mathrm{f}}^{\prime}\right\}_{x} \dot{w}_{1} \dot{w}_{2}\right)\right] \mathrm{d} z
\end{aligned}
$$


where primes denote differentiation with respect to $x$ and the terms in the braces represent a definite integration with respect to the variable denoted by the subscript, thus:

$$
\{F(x)\}_{x}=\int_{-b / 2}^{b / 2} F(x) \mathrm{d} x,
$$

where $F$ is an example function. The respective expanded expressions for the local bending strain energy stored in the more and less compressed webs are obtained in the same way and have a very similar format. The total local bending energy $U_{\mathrm{b}, \mathrm{l}}$ can be written as:

$$
U_{\mathrm{b}, \mathrm{l}}=U_{\mathrm{b}, \mathrm{f}}+U_{\mathrm{b}, \mathrm{wc}}+U_{\mathrm{b}, \mathrm{wt}} .
$$

\subsubsection{Membrane strain energy}

The membrane strain energy in the flanges $U_{\mathrm{m}, \mathrm{f}}$ is formulated by considering the direct strains $\left(\varepsilon_{z}\right)$ and the shear strains $(\gamma)$ in the flanges. The direct strains comprise three contributions, the first term from the global tilt mode and $u_{\mathrm{t}}=x \theta(z)$, the next two terms from the local mode obtained based on von Kármán plate theory, and finally a purely in-plane compressive strain term $\Delta$ :

$$
\begin{aligned}
\varepsilon_{z, \mathrm{f}}= & \frac{\partial u_{t}}{\partial z}+\frac{\partial u_{\mathrm{f}}}{\partial z}+\frac{1}{2}\left(\frac{\partial w_{\mathrm{f}}}{\partial z}\right)^{2}-\Delta \\
=- & x q_{t} \frac{\pi^{2}}{L} \sin \frac{\pi z}{L}+\left\{g_{1 \mathrm{f}}\right\}_{x} \dot{u}_{1}+\left\{g_{2 \mathrm{f}}\right\}_{x} \dot{u}_{2} \\
& \quad+\frac{1}{2}\left\{f_{1 \mathrm{f}}^{2}\right\}_{x} \dot{w}_{1}^{2}+\frac{1}{2}\left\{f_{2 \mathrm{f}}^{2}\right\}_{x} \dot{w}_{2}^{2}+\left\{f_{1 \mathrm{f}} f_{2 \mathrm{f}}\right\}_{x} \dot{w}_{1} \dot{w}_{2}-\Delta .
\end{aligned}
$$

The shear strain component can be written thus:

$$
\begin{aligned}
\gamma_{x z, \mathrm{f}}= & \frac{\partial u_{\mathrm{f}}}{\partial x}+\frac{\partial W}{\partial z}-\theta+\frac{\partial w_{\mathrm{f}}}{\partial x} \frac{\partial w_{\mathrm{f}}}{\partial z} \\
=\{ & \left.g_{1 \mathrm{f}}^{\prime}\right\}_{x} u_{1}+\left\{g_{2 \mathrm{f}}^{\prime}\right\}_{x} u_{2}-\left(q_{s}-q_{t}\right) \pi \cos \frac{\pi z}{L}+\left\{f_{1 \mathrm{f}}^{\prime} f_{1 \mathrm{f}}\right\}_{x} \dot{w}_{1} w_{1} \\
& \quad+\left\{f_{2 \mathrm{f}}^{\prime} f_{2 \mathrm{f}}\right\}_{x} \dot{w}_{2} w_{2}+\left\{f_{1 \mathrm{f}}^{\prime} f_{2 \mathrm{f}}\right\}_{x} w_{1} \dot{w}_{2}+\left\{f_{1 \mathrm{f}} f_{2 \mathrm{f}}^{\prime}\right\}_{x} \dot{w}_{1} w_{2} .
\end{aligned}
$$

The complete expression for the membrane strain energy stored in the flanges can be written thus if the transverse strain is assumed to be $\varepsilon_{x, \mathrm{f}}=-\nu \varepsilon_{z, \mathrm{f}}$ for simplicity:

$$
U_{\mathrm{m}, \mathrm{f}}=2 \int_{0}^{L} \int_{-t_{\mathrm{f}} / 2}^{t_{\mathrm{f}} / 2} \int_{-b / 2}^{b / 2} \frac{1}{2}\left(E \varepsilon_{z, \mathrm{f}}^{2}+G \gamma_{x z, \mathrm{f}}^{2}\right) \mathrm{d} x \mathrm{~d} y \mathrm{~d} z .
$$

The membrane strain energy in the webs also comprises direct and shear strain energy contributions. The complete expressions for the direct strain in the more compressed and less compressed webs are very similar to those for the flanges presented in Eq. (21):

$$
\begin{aligned}
\varepsilon_{z, \mathrm{wc}}= & \frac{\partial u_{\mathrm{t}, \mathrm{wc}}}{\partial z}+\frac{\partial u_{\mathrm{wc}}}{\partial z}+\frac{1}{2}\left(\frac{\partial w_{\mathrm{wc}}}{\partial z}\right)^{2}-\Delta \\
=- & q_{t} \frac{b \pi^{2}}{2 L} \sin \frac{\pi z}{L}+\left\{g_{1 \mathrm{wc}}\right\}_{y} \dot{u}_{1}+\left\{g_{2 \mathrm{wc}}\right\}_{y} \dot{u}_{2} \\
& \quad+\frac{1}{2}\left\{f_{1 \mathrm{wc}}^{2}\right\}_{y} \dot{w}_{1}^{2}+\frac{1}{2}\left\{f_{2 \mathrm{wc}}^{2}\right\}_{y} \dot{w}_{2}^{2}+\left\{f_{1 \mathrm{wc}} f_{2 \mathrm{wc}}\right\}_{y} \dot{w}_{1} \dot{w}_{2}-\Delta,
\end{aligned}
$$




$$
\begin{aligned}
\varepsilon_{z, \mathrm{wt}}= & \frac{\partial u_{\mathrm{t}, \mathrm{wt}}}{\partial z}+\frac{\partial u_{\mathrm{wt}}}{\partial z}+\frac{1}{2}\left(\frac{\partial w_{\mathrm{wt}}}{\partial z}\right)^{2}-\Delta \\
= & q_{t} \frac{b \pi^{2}}{2 L} \sin \frac{\pi z}{L}+\left\{g_{1 \mathrm{wt}}\right\}_{y} \dot{u}_{1}+\left\{g_{2 \mathrm{wt}}\right\}_{y} \dot{u}_{2} \\
& +\frac{1}{2}\left\{f_{1 \mathrm{wt}}^{2}\right\}_{y} \dot{w}_{1}^{2}+\frac{1}{2}\left\{f_{2 \mathrm{wt}}^{2}\right\}_{y} \dot{w}_{2}^{2}+\left\{f_{1 \mathrm{wt}} f_{2 \mathrm{wt}}\right\}_{y} \dot{w}_{1} \dot{w}_{2}-\Delta,
\end{aligned}
$$

where $u_{\mathrm{t}, \mathrm{wc}}=-b \theta(z) / 2$ and $u_{\mathrm{t}, \mathrm{wt}}=b \theta(z) / 2$ are axial displacements contributed by the tilt mode.

Unlike for the flanges, the shear strains within the webs only contain the terms from the local mode, hence:

$$
\begin{aligned}
\gamma_{y z, \mathrm{wc}}= & \frac{\partial u_{\mathrm{wc}}}{\partial y}+\frac{\partial w_{\mathrm{wc}}}{\partial y} \frac{\partial w_{\mathrm{wc}}}{\partial z} \\
=\{ & \left.g_{1 \mathrm{wc}}^{\prime}\right\}_{y} u_{1}+\left\{g_{2 \mathrm{wc}}^{\prime}\right\}_{y} u_{2}+\left\{f_{1 \mathrm{wc}}^{\prime} f_{1 \mathrm{wc}}\right\}_{y} \dot{w}_{1} w_{1}+\left\{f_{2 \mathrm{wc}}^{\prime} f_{2 \mathrm{wc}}\right\}_{y} \dot{w}_{2} w_{2} \\
& \quad+\left\{f_{1 \mathrm{wc}}^{\prime} f_{2 \mathrm{wc}}\right\}_{y} w_{1} \dot{w}_{2}+\left\{f_{1 \mathrm{wc}} f_{2 \mathrm{wc}}^{\prime}\right\}_{y} \dot{w}_{1} w_{2}, \\
\gamma_{y z, \mathrm{wt}}= & \frac{\partial u_{\mathrm{wt}}}{\partial y}+\frac{\partial w_{\mathrm{wt}}}{\partial y} \frac{\partial w_{\mathrm{wt}}}{\partial z} \\
= & \left\{g_{1 \mathrm{wt}}^{\prime}\right\}_{y} u_{1}+\left\{g_{2 \mathrm{wt}}^{\prime}\right\}_{y} u_{2}+\left\{f_{1 \mathrm{wt}}^{\prime} f_{1 \mathrm{wt}}\right\}_{y} \dot{w}_{1} w_{1}+\left\{f_{2 \mathrm{wt}}^{\prime} f_{2 \mathrm{wt}}\right\}_{y} \dot{w}_{2} w_{2} \\
& \quad+\left\{f_{1 \mathrm{wt}}^{\prime} f_{2 \mathrm{wt}}\right\}_{y} w_{1} \dot{w}_{2}+\left\{f_{1 \mathrm{wt}} f_{2 \mathrm{wt}}^{\prime}\right\}_{y} \dot{w}_{1} w_{2} .
\end{aligned}
$$

Again, assuming that $\varepsilon_{y, \mathrm{wc}}=-\nu \varepsilon_{z \text {,wc }}$ and $\varepsilon_{y, \mathrm{wt}}=-\nu \varepsilon_{z, \mathrm{wt}}$, the membrane strain energy contributions from both webs are given thus:

$$
U_{\mathrm{m}, \mathrm{w}}=\frac{1}{2} \int_{0}^{L} \int_{-d / 2}^{d / 2} \int_{-t_{\mathrm{w}} / 2}^{t_{\mathrm{w}} / 2}\left[E\left(\varepsilon_{z, \mathrm{wc}}^{2}+\varepsilon_{z, \mathrm{wt}}^{2}\right)+G\left(\gamma_{y z, \mathrm{wc}}^{2}+\gamma_{y z, \mathrm{wt}}^{2}\right)\right] \mathrm{d} x \mathrm{~d} y \mathrm{~d} z .
$$

\subsubsection{Work done by the load and total potential energy}

The total end-shortening, $\mathcal{E}$, comprises terms from pure squash, the global sway mode and the local in-plane displacement. The work done by the external load is given by:

$$
P \mathcal{E}=P \int_{0}^{L}\left[\Delta+q_{s}^{2} \frac{\pi^{2}}{2} \cos ^{2} \frac{\pi z}{L}-\Delta_{\mathrm{m}}\right] \mathrm{d} z
$$

where:

$$
\Delta_{\mathrm{m}}=\frac{\left(2 \phi_{\mathrm{t}}\left\{g_{1 \mathrm{f}}\right\}_{x}+\left\{g_{1 \mathrm{wc}}\right\}_{y}+\left\{g_{1 \mathrm{wt}}\right\}_{y}\right) \dot{u}_{1}+\left(2 \phi_{\mathrm{t}}\left\{g_{2 \mathrm{f}}\right\}_{x}+\left\{g_{2 \mathrm{wc}}\right\}_{y}+\left\{g_{2 \mathrm{wt}}\right\}_{y}\right) \dot{u}_{2}}{2 b\left(\phi_{\mathrm{t}}+\phi_{\mathrm{c}}\right)}
$$

The total potential energy $V$ can be expressed by the summation of all the strain energy terms minus the work done by the external load:

$$
V=U_{\mathrm{b}, \mathrm{o}}+U_{\mathrm{b}, \mathrm{l}}+U_{\mathrm{m}, \mathrm{f}}+U_{\mathrm{m}, \mathrm{w}}-P \mathcal{E}
$$




\subsubsection{Equilibrium equations}

By performing the calculus of variations on the total potential energy, the governing equilibrium equations can be obtained. The integrand of the total potential energy $V$ can be written as a Lagrangian $(\mathcal{L})$ of the form:

$$
V=\int_{0}^{L} \mathcal{L}\left(\ddot{w}_{i}, \dot{w}_{i}, w_{i}, \dot{u}_{i}, u_{i}, z\right) \mathrm{d} z,
$$

where $i=\{1,2\}$. The equilibrium states of the system can be obtained by determining the first variation of $V$ :

$$
\delta V=\int_{0}^{L}\left[\frac{\partial \mathcal{L}}{\partial \ddot{w}_{i}} \delta \ddot{w}_{i}+\frac{\partial \mathcal{L}}{\partial \dot{w}_{i}} \delta \dot{w}_{i}+\frac{\partial \mathcal{L}}{\partial w_{i}} \delta w_{i}+\frac{\partial \mathcal{L}}{\partial \dot{u}_{i}} \delta \dot{u}_{i}+\frac{\partial \mathcal{L}}{\partial u_{i}} \delta u_{i}\right] \mathrm{d} z
$$

and finding the conditions where $\delta V=0$. Since $\delta \ddot{w}_{i}=\mathrm{d}\left(\delta \dot{w}_{i}\right) / \mathrm{d} z, \delta \dot{w}_{i}=\mathrm{d}\left(\delta w_{i}\right) / \mathrm{d} z$ and $\delta \dot{u}_{i}=\mathrm{d}\left(\delta u_{i}\right) / \mathrm{d} z$, integration by parts allows the development of the Euler-Lagrange equations for $w_{i}$ and $u_{i}$, which comprise a fourth order nonlinear ordinary differential equation (ODE) for $w_{i}$ and second order nonlinear ODE for $u_{i}$, thus:

$$
\begin{array}{r}
\frac{\mathrm{d}^{2}}{\mathrm{~d} z^{2}}\left(\frac{\partial \mathcal{L}}{\partial \ddot{w}_{i}}\right)-\frac{\mathrm{d}}{\mathrm{d} z}\left(\frac{\partial \mathcal{L}}{\partial \dot{w}_{i}}\right)+\frac{\partial \mathcal{L}}{\partial w_{i}}=0, \\
\frac{\mathrm{d}}{\mathrm{d} z}\left(\frac{\partial \mathcal{L}}{\partial \dot{u}_{i}}\right)-\frac{\partial \mathcal{L}}{\partial u_{i}}=0 .
\end{array}
$$

Moreover, equilibrium also requires the minimization of $V$ with respect to the generalized coordinates $q_{s}, q_{t}$ and $\Delta$, thus:

$$
\frac{\partial V}{\partial q_{s}}=0, \quad \frac{\partial V}{\partial q_{t}}=0, \quad \frac{\partial V}{\partial \Delta}=0,
$$

which lead to three integral equations. The first expression in Eq. (36) provides a relationship between $q_{s}$ and $q_{t}$ before the local mode is triggered, i.e. when $u_{i}, w_{i}$ and their derivatives with respect to $z$ are zero:

$$
q_{s}=(1+s) q_{t},
$$

where:

$$
s=\frac{\pi^{2} E b^{2}}{12 G L^{2}}\left(1+\frac{3 \phi_{\mathrm{c}}}{\phi_{\mathrm{t}}}\right) .
$$

The boundary conditions for $w_{i}, u_{i}$ and their derivatives are for simply-supported conditions at $z=0$ and for symmetry conditions at $z=L / 2$ :

$$
w_{i}(0)=\ddot{w}_{i}(0)=\dot{w}_{i}(L / 2)=\dddot{w}_{i}(L / 2)=u_{i}(L / 2)=0 .
$$

Two further boundary conditions can be obtained from the variational formulation with regards to the in-plane displacements $u_{i}$, hence:

$$
\left[\frac{\partial \mathcal{L}}{\partial \dot{u}_{i}} \delta u_{i}\right]_{0}^{L}=0 .
$$


Linear eigenvalue analysis for the perfect strut is conducted to determine the global buckling load $P_{\mathrm{o}}^{\mathrm{C}}$. The criticality condition for equilibrium is invoked by considering where the Hessian matrix of the total potential energy $V$ is singular on the fundamental path $\mathrm{F}$, where $q_{s}=q_{t}=w_{i}=u_{i}=0$, hence:

$$
\operatorname{det}\left(\mathbf{V}_{i j}^{\mathrm{C}}\right)=\left|\begin{array}{cc}
\frac{\partial^{2} V}{\partial q_{s}^{2}} & \frac{\partial^{2} V}{\partial q_{s} \partial q_{t}} \\
\frac{\partial^{2} V}{\partial q_{t} \partial q_{s}} & \frac{\partial^{2} V}{\partial q_{t}^{2}}
\end{array}\right|^{\mathrm{F}}=0 .
$$

Solving Eq. (41) gives the following expression:

$$
P_{\mathrm{o}}^{\mathrm{C}}=\frac{2 \pi^{2} E I_{\mathrm{w}}}{L^{2}}+\frac{\pi^{2} E t_{\mathrm{f}} b^{3}}{6(1+s) L^{2}}\left(1+\frac{3 \phi_{\mathrm{c}}}{\phi_{\mathrm{t}}}\right)
$$

Note that if Euler-Bernoulli bending theory had been assumed, the shear modulus $G \rightarrow \infty$ and hence $s \rightarrow 0$, then the expression for $P_{\mathrm{o}}^{\mathrm{C}}$ would reduce to the classical Euler load, as would be expected.

\subsection{Numerical solution strategy for variational model}

Since the complete system of the coupled nonlinear ordinary differential equations is too complicated to be solved analytically, it is therefore solved numerically using the continuation and bifurcation software AUTO-07P [62]. This software has been shown in previous studies [56, 57, 12, 13, 58, 42] to be capable of solving nonlinear differential equations without losing the intrinsic mathematical structure of the solutions and of switching between, as well as tracing, different equilibrium paths.

According to the previous studies on mode interaction in thin-walled compression members [8, 29, 26, 27, 44], the behaviour can be classified into four distinct zones that are related to the strut length $L$. Currently, these have been assigned the qualitative names 'long', 'transitional', 'intermediate' and 'short' length struts and respectively correspond to the zones labelled 1-4 shown in Figure 1(b). The different equilibrium diagrams alongside the numerical continuation procedures to compute the equilibrium paths of the four representative struts using AUTO are shown diagrammatically in Figure 5. Figure 5(a) depicts the equilibrium behaviour for the case where global buckling is critical and was described in detail in [52]. The critical buckling load $P_{\mathrm{o}}^{\mathrm{C}}$ at the critical bifurcation point $\mathrm{C}$ is obtained explicitly from Eq. (42). Using the continuation method, the generalized coordinate of the global sway mode $q_{s}$ is first varied with the load $P=P_{\mathrm{o}}^{\mathrm{C}}$ to obtain the secondary bifurcation point $\mathrm{S}$, where interactive buckling is triggered. Subsequently, using the branch switching facility in AuTO, the second run is started at the secondary bifurcation point $\mathrm{S}$ and the load $P$ is varied to compute the interactive buckling path.

However, for the cases where local buckling is critical, i.e. for zones 2-4, as presented in Figure 1(b), there is known to be some numerical difficulty in switching from the pure local buckling path to the interactive buckling path [13, 28, 63]. Therefore, the numerical continuation (so-called 'homotopy') strategy is adopted to obtain the solution, as described in Figure 6. The solution begins from determining the equilibrium path for a strut with 
(a) Long

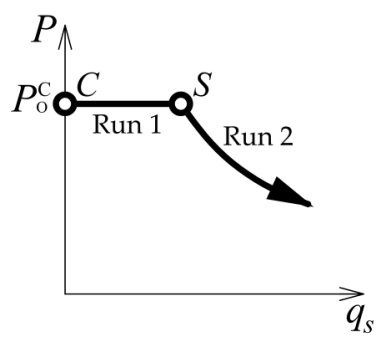

(b) Transitional

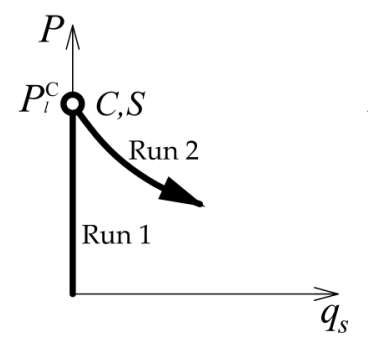

(c) Intermediate

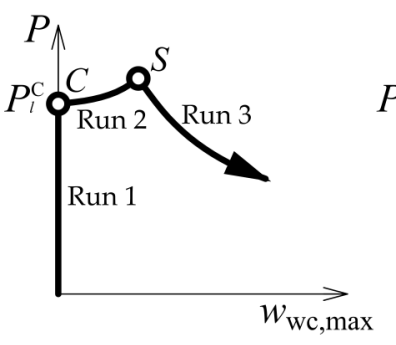

(d) Short

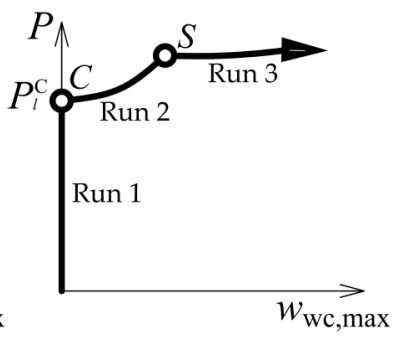

Figure 5: Sketches of the equilibrium paths for struts of different lengths exhibiting mode interaction. Graphs (a)-(d) correspond to the zones labelled 1-4 as presented in the van der Neut curve in Figure 1(b). Circles marked $\mathrm{C}$ and $\mathrm{S}$ represent the critical and secondary bifurcation points for each case respectively. Note that the abscissa changes from $q_{s}$ in $(\mathrm{a}-\mathrm{b})$ to $w_{\mathrm{wc}, \max }$ in $(\mathrm{c}-\mathrm{d})$, which reflects the appropriate measure of the critical buckling mode.

length $L_{1}$, a case where global buckling is critical, referring to Runs 1 and 2 depicted in Figure 6. Then by changing the principal varying parameter to the strut length $L$ in AuTO, the interactive buckling equilibrium states of struts with different lengths can be obtained, referring to Run 3, depicted in Figure 6] which begins at point $R_{1}$. The interactive buckling path of the strut with the new length $L_{2}$, where local buckling is critical, can be computed from the point $\mathrm{R}_{2}$ by re-setting the principal varying parameter to $q_{s}$. The solution of this branch ends at the new secondary bifurcation point $\mathrm{S}_{2}$.

As for the fundamental and pure local buckling equilibrium path, as shown in Run 1 in Figure 5(b) and Runs 1 and 2 in Figure $5(\mathrm{c}-\mathrm{d})$, the continuation process initiates from zero load and the local buckling load $P_{1}^{\mathrm{C}}$ is obtained numerically. The post-buckling path is then computed by using the branch switching facility and the local buckling equilibrium path ends at the secondary bifurcation point $\mathrm{S}$, where critical local buckling is initially destabilized due to contamination from the global buckling mode.

\subsection{FE model for validation}

For validation purposes, nonlinear FE models of the example struts with the same material and geometric properties as the variational model are developed in the commercial FE package ABAQUS [53] using four-noded, reduced-integration S4R shell elements. Owing to the discontinuous pitchfork bifurcation response at the point of initial instability, it is not possible to analyse the perfect post-buckling behaviour in ABAQUS directly. Therefore, by introducing an initial perturbation in the geometry, the discontinuous bifurcation problem is necessarily avoided. In the current study, the eigenmodes from linear buckling analysis are adopted as the profiles for the initial global and local geometric perturbations and the scale size for the global and local perturbations are set to $10^{-6} \mathrm{~L}$ and $10^{-3} t_{\mathrm{w}}$ respectively. These sufficiently small magnitudes ensure that the response essentially mimics the perfect system as far as possible, without encountering the convergence problems caused by the equilibrium path discontinuity from the bifurcations. As for the nonlinear solution, the 

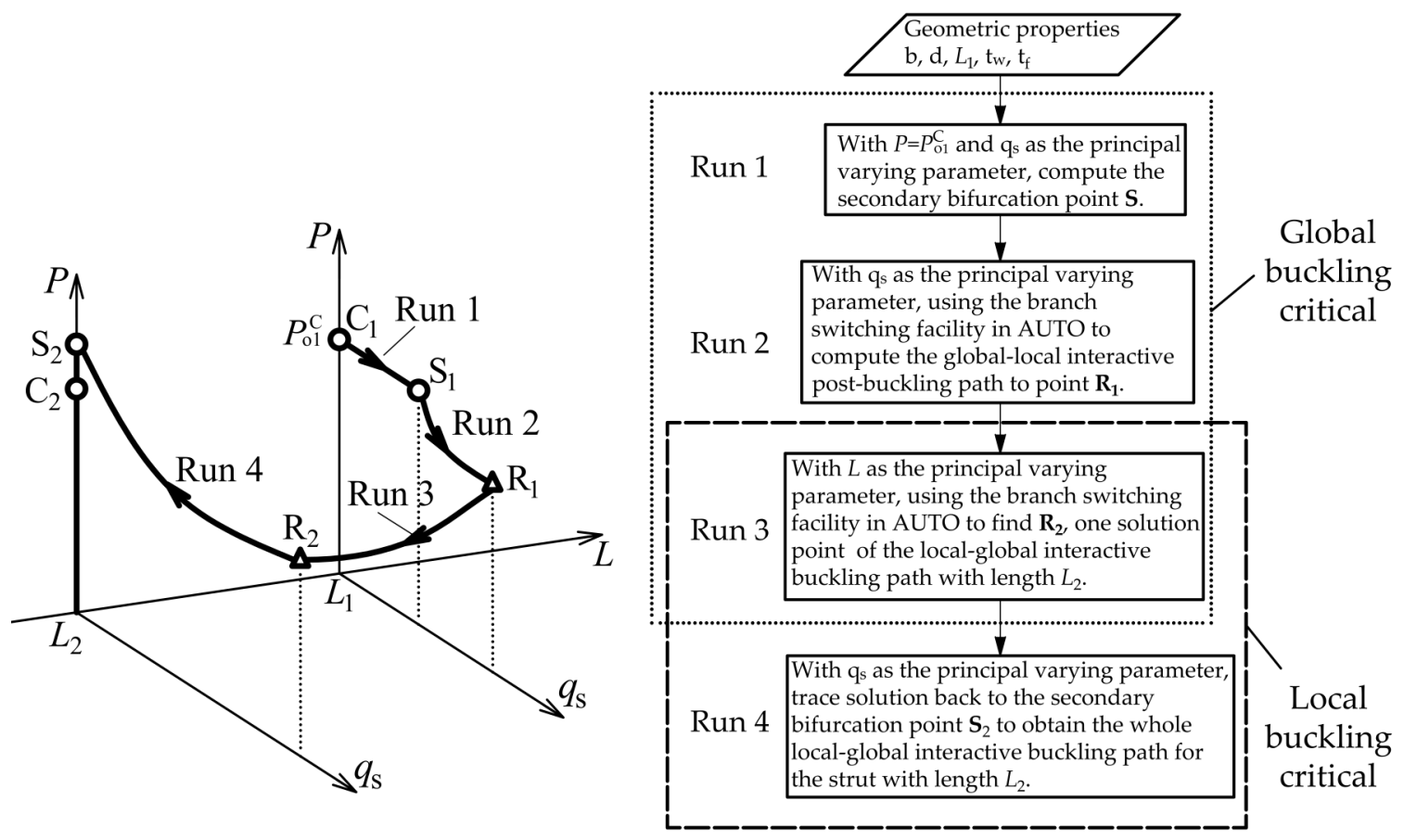

Figure 6: Sketch and flowchart of the numerical continuation procedure for determining the interactive buckling equilibrium path for perfect struts where local buckling is critical. Circles marked $\mathrm{C}_{i}$ and $\mathrm{S}_{i}$ represent the critical and secondary bifurcation points respectively; triangles marked $\mathrm{R}_{i}$ represent generic equilibrium states on the interactive buckling paths with strut lengths $L_{i}$. Strut lengths $L_{1}$ and $L_{2}$ represent the cases where global buckling and local buckling are critical respectively; $P_{\mathrm{o} 1}^{\mathrm{C}}$ is the global buckling load of the strut with length $L_{1}$.

Riks arclength method [64] is adopted to trace the nonlinear post-buckling equilibrium path. The full modelling details and analysis procedures can be found in [52].

\section{Numerical results and validation}

Four representative numerical examples from the variational model with the same crosssection properties but different lengths are presented. The cross-section geometric and material properties of the example struts are presented in Table 1 with Table 2 summarizing the different lengths, the critical buckling loads and corresponding zones of the example struts.

\subsection{Long length strut}

The interactive buckling behaviour of long struts has been investigated in [52], which showed excellent comparisons with the FE model. For the present long length strut, where $L=4800 \mathrm{~mm}$, a sharp snap-back in the load-end-shortening relationship is observed, as shown in Figure 7 (a). Severely imperfection sensitive behaviour would be expected for such a strut with $P_{\mathrm{o}}^{\mathrm{C}} / P_{1}^{\mathrm{C}}$ being close to unity [25, 26]. 
(a)

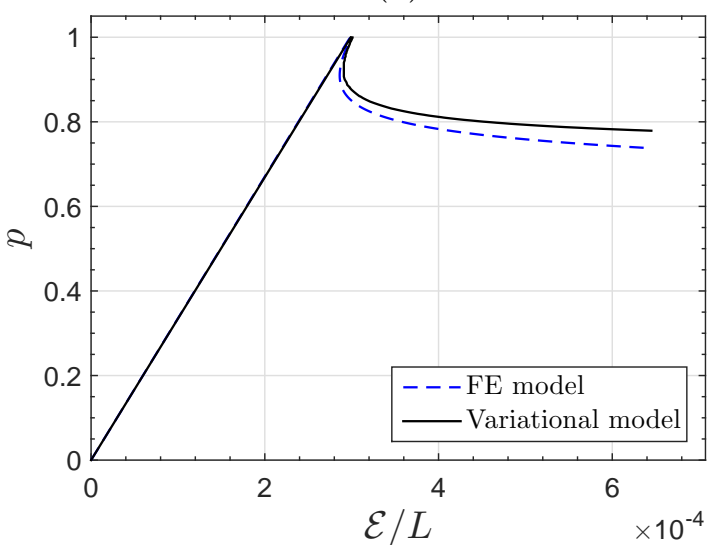

(c)



(b)

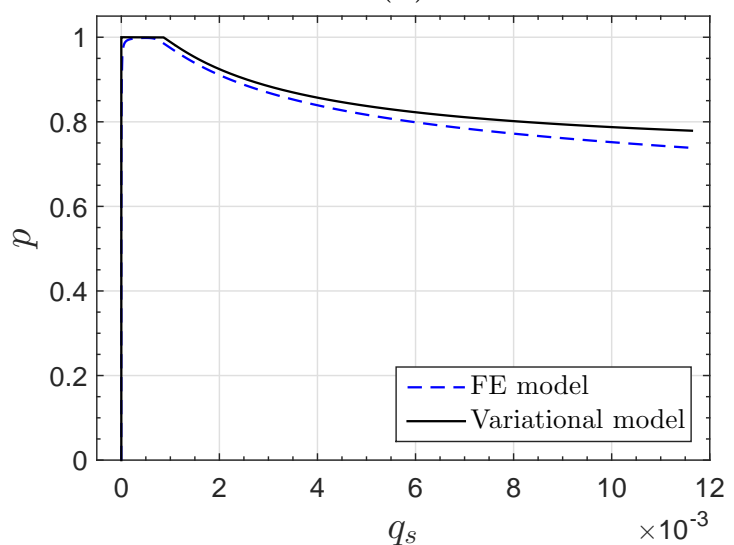

(d)



Figure 7: Nonlinear equilibrium paths for the example long length strut with $L=4800 \mathrm{~mm}$ from the variational and FE models. Graphs of the normalized load ratio $p=P / P^{\mathrm{C}}$, where $P^{\mathrm{C}}$ is the critical buckling load, versus (a) the normalized end-shortening $\mathcal{E} / L$, (b) the generalized coordinate of the sway mode $q_{s}$, and (c) the normalized maximum amplitude of the local buckling deflection in the more compressed web $w_{\mathrm{wc}, \max } / t_{\mathrm{w}} ;(\mathrm{d}) w_{\mathrm{wc}, \max } / t_{\mathrm{w}}$ versus $q_{s}$. 
Table 1: Cross-section and material properties of the rectangular hollow section struts in the numerical examples.

\begin{tabular}{ll}
\hline Flange width $b$ & $60 \mathrm{~mm}$ \\
Web depth $d$ & $120 \mathrm{~mm}$ \\
Flange thickness $t_{\mathrm{f}}$ & $1 \mathrm{~mm}$ \\
Web thickness $t_{\mathrm{w}}$ & $1 \mathrm{~mm}$ \\
Young's Modulus $E$ & $210 \mathrm{kN} / \mathrm{mm}^{2}$ \\
Poisson's ratio $\nu$ & 0.3 \\
\hline
\end{tabular}

Table 2: Theoretical values of the global $\left(P_{\mathrm{o}}^{\mathrm{C}}\right)$ and local $\left(P_{1}^{\mathrm{C}}\right)$ buckling loads for the four representative length cases.

\begin{tabular}{ccccccc}
\hline$L(\mathrm{~mm})$ & $P_{\mathrm{o}}^{\mathrm{C}}(\mathrm{kN})$ & $P_{1, \mathrm{AutO}}^{\mathrm{C}}(\mathrm{kN})$ & $P_{1, \mathrm{ABAQUS}}^{\mathrm{C}}(\mathrm{kN})$ & $P_{\mathrm{o}}^{\mathrm{C}} / P_{1}^{\mathrm{C}}$ & Zone & Length description \\
\hline 4800 & 22.67 & 24.61 & 24.57 & 0.92 & 1 & 'Long' \\
4500 & 25.79 & 24.61 & 24.58 & 1.05 & 2 & 'Transitional' \\
4000 & 32.54 & 24.57 & 24.58 & 1.32 & 3 & 'Intermediate' \\
3600 & 40.30 & 24.67 & 24.58 & 1.63 & 4 & 'Short' \\
\hline
\end{tabular}

From the solutions of the out-of-plane components of the local mode in the more compressed web $w_{\mathrm{wc}}$, the increase of the global buckling mode amplitude not only leads to an increase in the local mode amplitude, as shown in Figure $\mathbf{7}(\mathrm{d})$, but also forces a change of the local buckling profile from being localized to being more distributed alongside a corresponding reduction in the local buckling mode wavelength, as shown in Figure 8 .

Compared with the FE model, the variational model shows a slightly stiffer postbuckling response, as shown in Figure [7. One of the sources for the stiffer response is derived from the assumption that the location of the neutral axis remains unchanged in the variational model. In fact, the neutral axis would move towards the less compressed web as the effective stiffness of the more compressed web drops due to it buckling locally. Moreover, in the variational model, the cross-section profile of the local mode is assumed to be the same along the length of strut and throughout the post-buckling range with the only variable being the modal amplitude of local buckling. However, the profile is in fact affected by the bending moment and axial stress on the cross-section, which varies along the strut length and with the progression of mode interaction. This may explain the small discrepancy in the longitudinal solutions of the local deflection amplitude in the more compressed web $w_{\mathrm{wc}} / t_{\mathrm{w}}$, as shown in Figure 8, and the 'bulging-out' effect due to the high axial stress in the more compressed web in Figure 9(d). It should be mentioned that there is a tiny out-of-plane displacement in the less compressed web in the initial post-buckling stage of the FE results, as shown in Figure 8( $(\mathrm{e}-\mathrm{h})$ and Figure 9(a). With the increase of the global mode amplitude, the change in the profile of the local mode nearly follows the same pattern as that in the more compressed web, i.e. from localized to more distributed, but its amplitude remains approximately the same. The kinematic compatibility between the buckled flanges and the less compressed web leads to the tiny deformation. When the generalized coordinate of the global mode $q_{s}$ is relatively larger, the entirety of the 
(a) $q_{s}=0.001$

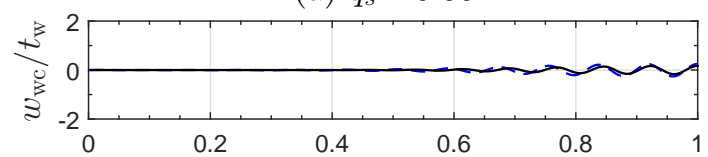

(b) $q_{s}=0.002$

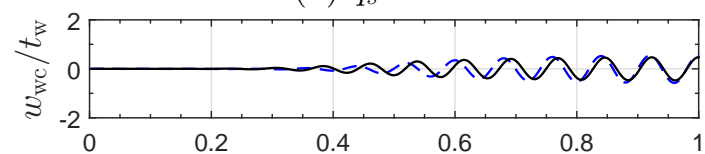

(c) $q_{s}=0.004$

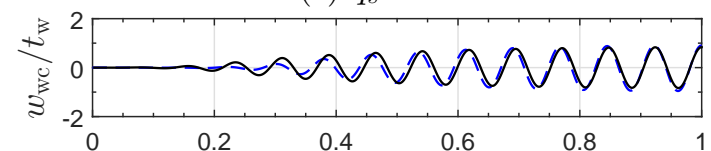

(d) $q_{s}=0.010$

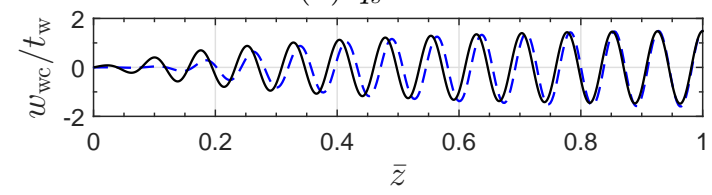

(e) $q_{s}=0.001$

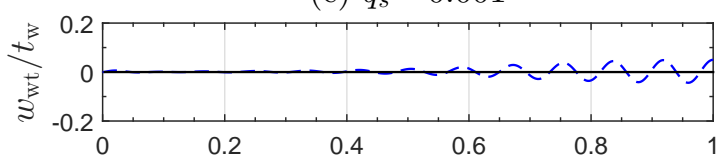

(f) $q_{s}=0.002$

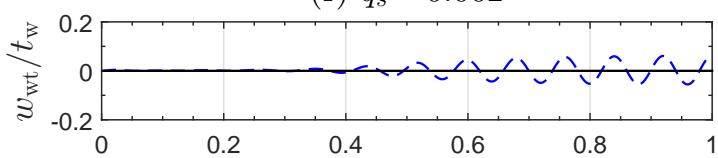

(g) $q_{s}=0.004$

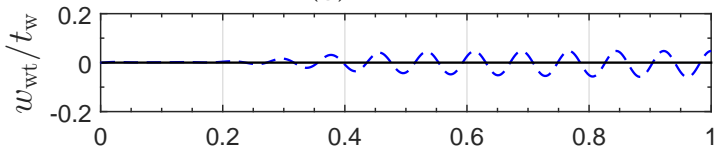

(h) $q_{s}=0.010$

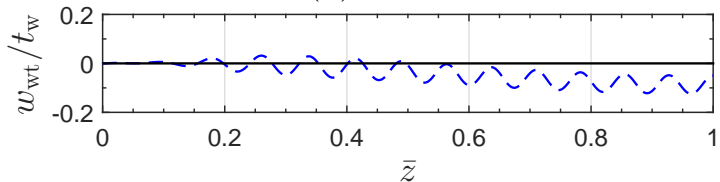

Figure 8: Evolution of the numerical solutions for the normalized local out-of-plane displacement in (a-d) the more compressed web $w_{\mathrm{wc}} / t_{\mathrm{w}}$ and $(\mathrm{e}-\mathrm{h})$ the less compressed web $w_{\mathrm{wt}} / t_{\mathrm{w}}$ for the long length strut with $L=4800 \mathrm{~mm}$. The dashed and solid lines represent the numerical results from the FE and the variational model respectively. Note that the longitudinal coordinate is normalized with respect to half of the strut length $\bar{z}=2 z / L$ and that $w_{\mathrm{wc}} / t_{\mathrm{w}}$ is an order of magnitude greater than $w_{\mathrm{wt}} / t_{\mathrm{w}}$.

(a) $q_{s}=0.001$

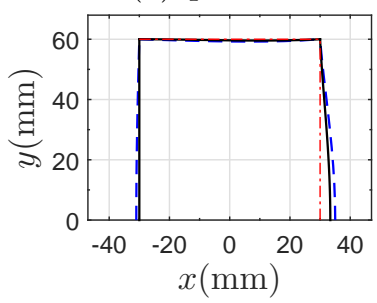

(b) $q_{s}=0.004$

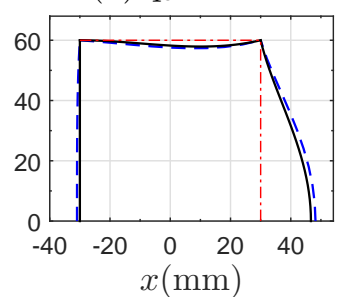

(c) $q_{s}=0.006$



(d) $q_{s}=0.010$

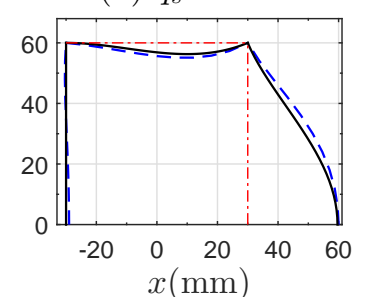

Figure 9: Local deformation of the cross-section at mid-span for the long length strut with $L=4800$ $\mathrm{mm}$ with the increase of the generalized sway mode coordinate $q_{s}$. The dashed and solid lines represent the numerical results from the FE and variational models respectively. The dot-dashed line represents the undeformed shape. Note that the displacements shown have been amplified by a factor of 20 to aid visualization. 
less compressed web deforms inwards slightly, as shown in Figures $8(\mathrm{~h})$ and $9(\mathrm{~d})$. This is caused by the out-of-plane force introduced by the global mode that is identical to the phenomenon that causes the 'Brazier effect' in cylindrical shells under uniform bending [65, 66], as shown in Figure [0, which is not included in the current variational model. All

(a)

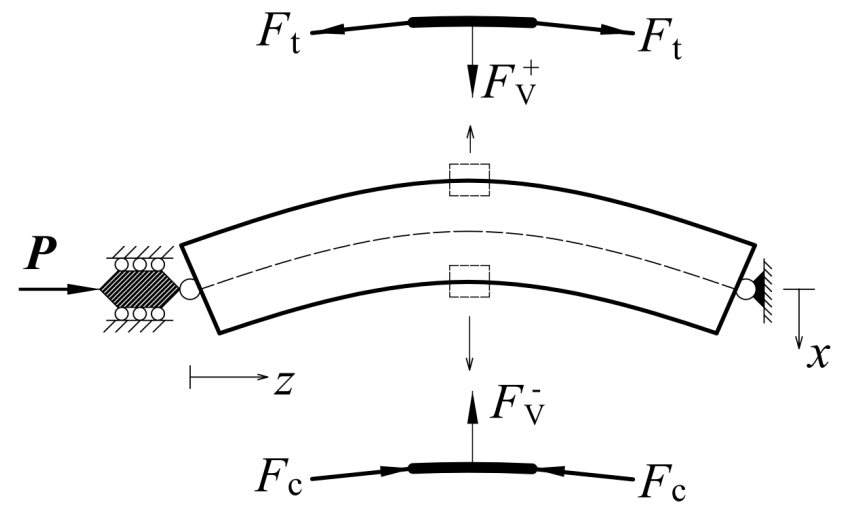

(b)

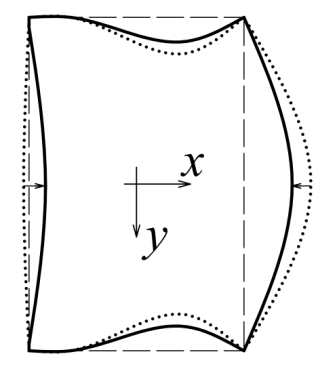

Figure 10: (a) The out-of-plane forces due to the large bending displacement with $F_{\mathrm{V}}$ representing the effective vertical component, as shown, of the axial force within each web $\left(F_{\mathrm{c}}\right.$ or $\left.F_{\mathrm{t}}\right)$. (b) The corresponding effects on the local mode profile - the dotted line and the thick solid line represent the cross-section profile before and after considering the global bending effects respectively.

of these factors taken together lead to the very marginally stiffer response in the variational model for the long strut, but it is, in fact, very small.

\subsection{Transitional length strut}

For the transitional length strut considered, where $L=4500 \mathrm{~mm}$, an even more severe snap-back is observed in the load-end-shortening relationship, as shown in Figure 11(a). This is caused by the fact that when local buckling initially occurs, the actual stiffness of the strut is reduced, effectively leading to a reduced global buckling load, which becomes instantaneously smaller than the critical local buckling load $P_{1}^{\mathrm{C}}$. Hence, both buckling modes are effectively triggered simultaneously, as shown in Figure 11(d).

The solutions of the local deflection in the more and less compressed webs are shown in Figure 12. The amplitude in the more compressed web increases with the progression of mode interaction, while the amplitude in the less compressed web remains approximately unchanged. This leads to a transition of the cross-section deformation from being close to the pure local mode (symmetrical about the $y$-axis) to being dominated by the global mode (asymmetrical about the $y$-axis), as shown in Figure 13. Since local buckling is critical for the transitional length strut, the longitudinal profile of the local mode is initially distributed rather than localized. However, a reduction in the local buckling wavelength with the increase of the global mode amplitude is also observed as the axial end-shortening is increased. 
(a)

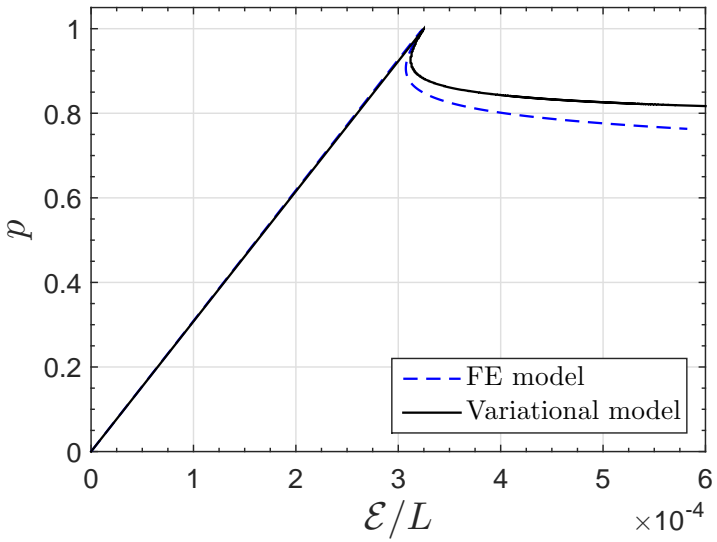

(c)

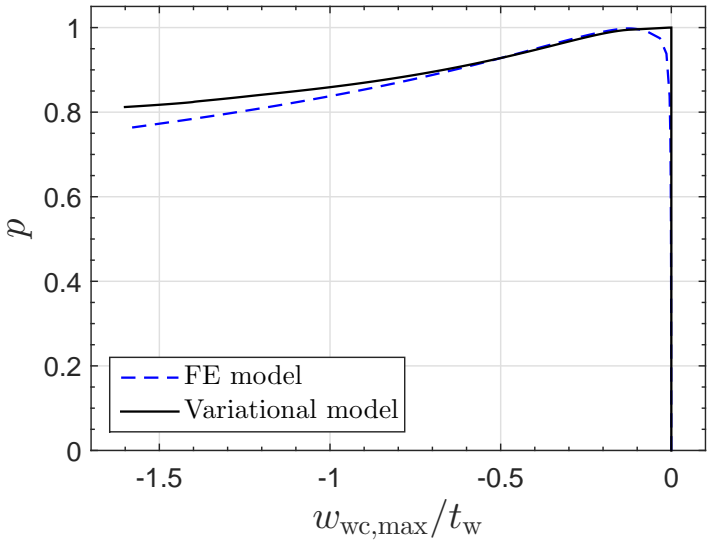

(b)

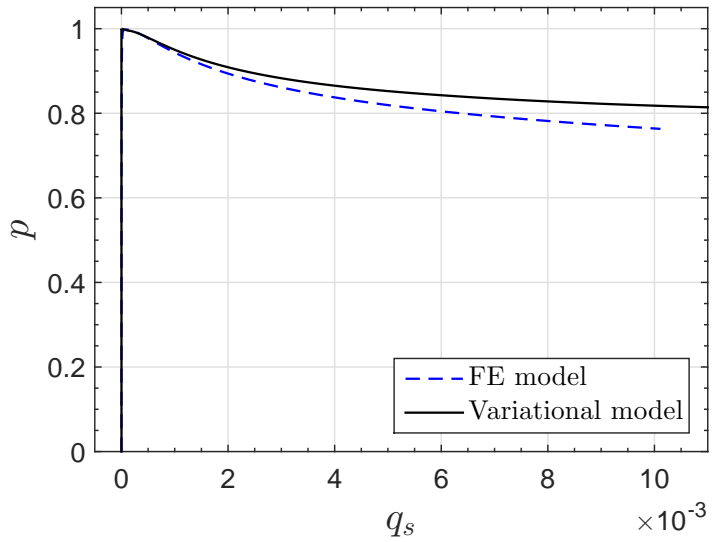

(d)

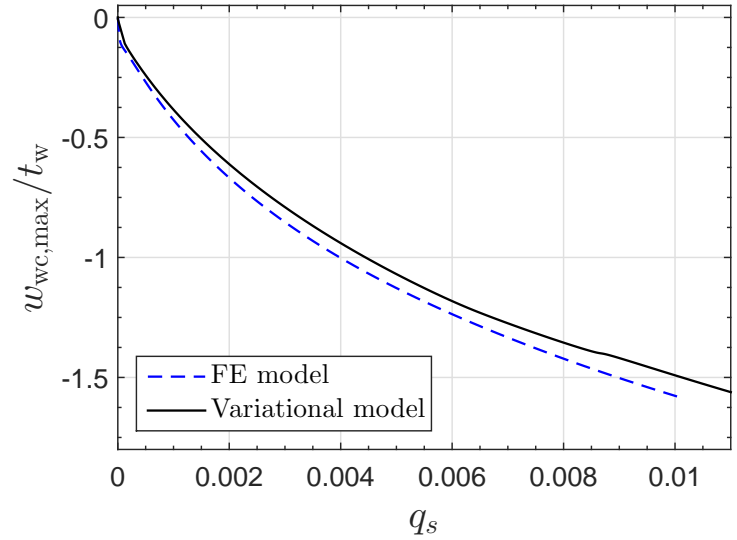

Figure 11: Nonlinear equilibrium paths for the transitional length strut with $L=4500 \mathrm{~mm}$ from the variational and FE models. Graphs (a)-(d) are as described in Figure 7

The variational model for the transitional length strut also shows a stiffer post-buckling response when compared to the corresponding FE model, as can be seen from the equilibrium path comparison in Figure 11. The reasons listed for the long strut also apply presently. The excellent comparisons between the solution of the out-of-plane displacement in the more compressed web and the cross-section deformation, particularly when $q_{s}$ is relatively small, validate the current methodology. As shown in Figure 10, with the increase of the global buckling amplitude, the less compressed web bulges inwards. It can be seen clearly from the solution of the out-of-plane displacement in the less compressed web from the FE model in Figure 12(g, h), where the solution wave drifts to the negative direction. This can also help to explain the discrepancy in the cross-section deformation for the less compressed web at mid-span when $q_{s}$ is large, as shown in Figure 13 (c-d). 
(a) $q_{s}=0.0005$

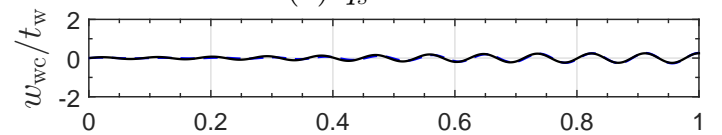

(b) $q_{s}=0.001$

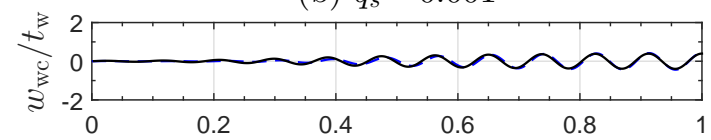

(c) $q_{s}=0.005$

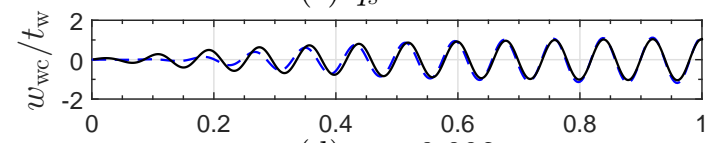

(d) $q_{s}=0.008$

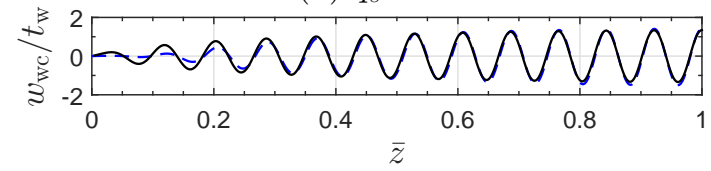

(e) $q_{s}=0.0005$

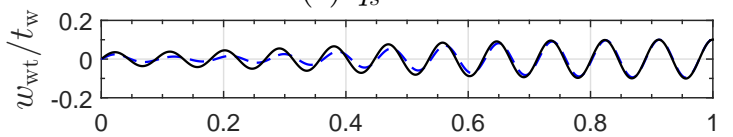

(f) $q_{s}=0.001$

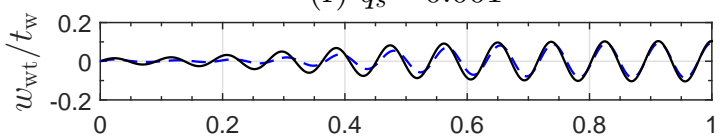

(g) $q_{s}=0.005$

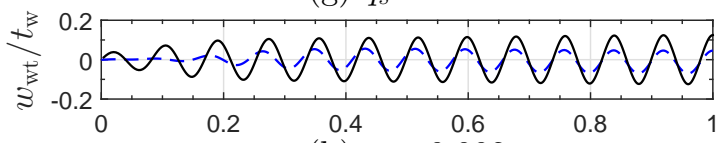

(h) $q_{s}=0.008$

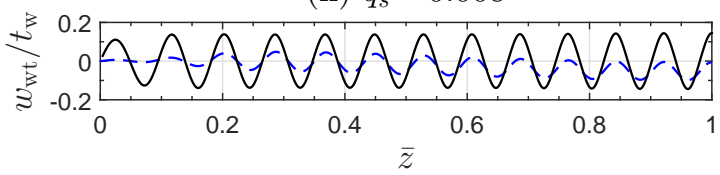

Figure 12: Evolution of the numerical solutions for $(\mathrm{a}-\mathrm{d}) w_{\mathrm{wc}} / t_{\mathrm{w}}$ and $(\mathrm{e}-\mathrm{h}) w_{\mathrm{wt}} / t_{\mathrm{w}}$ for the transitional length strut with $L=4500 \mathrm{~mm}$. The graphs are as described in Figure 8

(a) $q_{s}=0.0005$

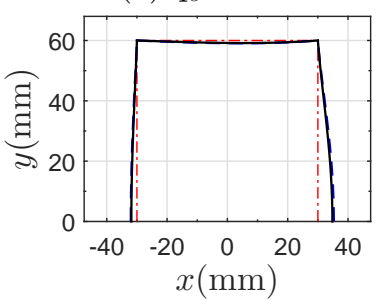

(b) $q_{s}=0.001$

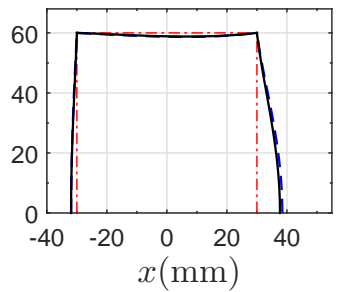

(c) $q_{s}=0.005$

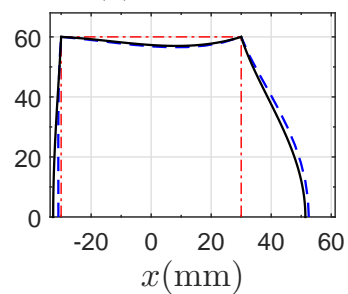

(d) $q_{s}=0.008$



Figure 13: Local deformation of the cross-section at mid-span for the transitional length strut with $L=$ $4500 \mathrm{~mm}$ as $q_{s}$ is increased. The graphs are as described in Figure 9

\subsection{Intermediate length strut}

For the intermediate length strut, where $L=4000 \mathrm{~mm}$, there are three stages in the equilibrium paths for the load versus end-shortening and versus the local buckling amplitude, as shown in Figure 14(a, c). The first stage involves purely axial deformation of the strut before the critical buckling load $P_{1}^{\mathrm{C}}$ is reached $(p<1)$. The second stage is the local buckling of the whole cross-section and the third stage is the local-global mode interaction. Before the secondary bifurcation, the strut is stable; however, the triggering of the global mode leads to unstable post-buckling behaviour. In the interactive buckling range, there are several snap-backs in the equilibrium path, which correspond to the local mode increasing its number of peaks hence reducing its wavelength, as shown in Figure 15 . This type of cellular buckling [43] was also observed in previous studies on I-section beams 12], I-section struts [13] and stiffened panels [14].

From the solutions of the out-of-plane displacements in the more and less compressed webs, the progression of mode interaction forces a reduction in wavelength. Since the local 
(a)

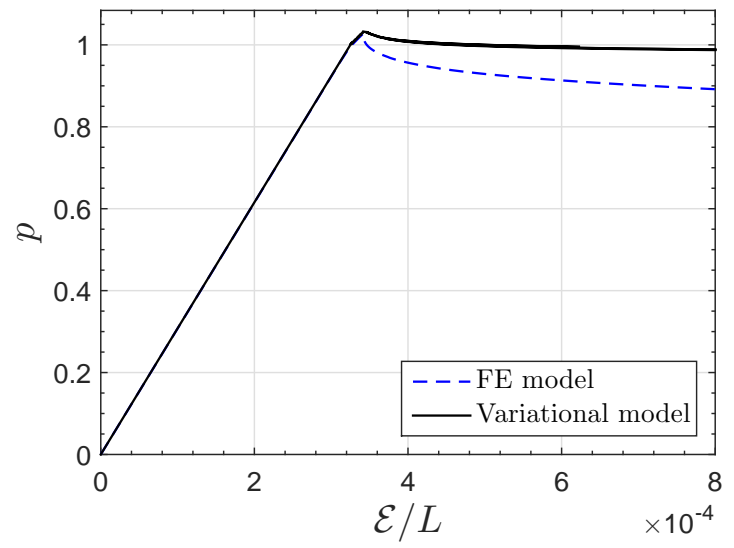

(c)

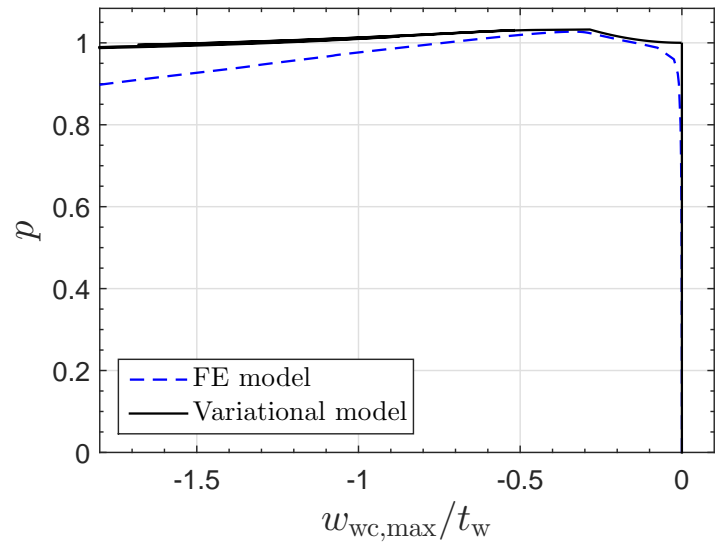

(b)

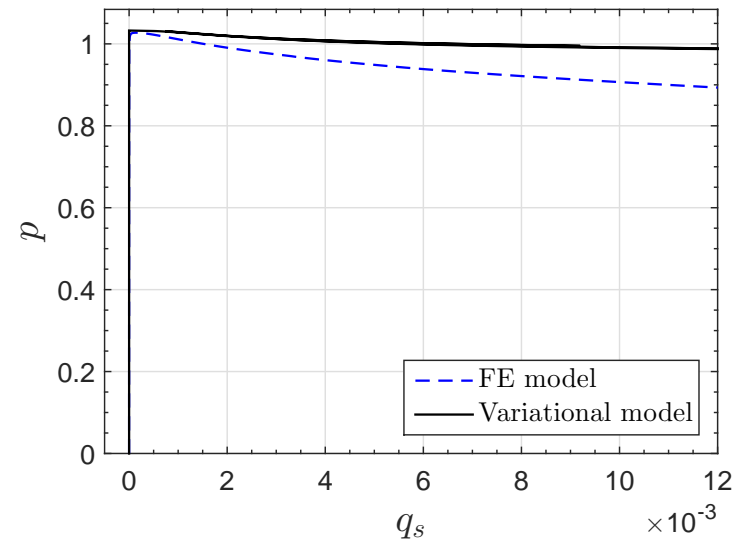

(d)

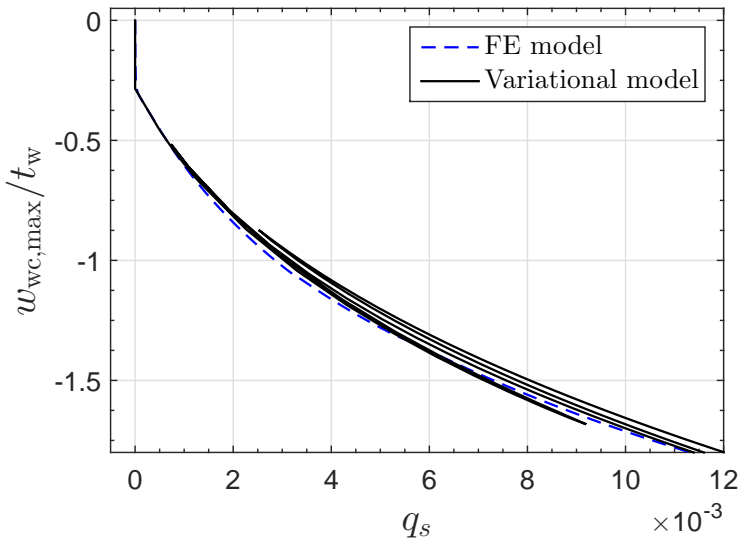

Figure 14: Nonlinear equilibrium paths for the intermediate length strut with $L=4000 \mathrm{~mm}$ from the variational and FE models. Graphs (a)-(d) are as described in Figure 7

buckling mode is well developed before the mode interaction, the wavelength reduction leads to an increase in the number of peaks and troughs, as shown in Figure 15, which is not observed in the previous two relatively slender example struts. Moreover, the displacement amplitude increases at mid-span but remains approximately unchanged at the ends, leading to the profile changing from periodic to being more localized. The mode interaction makes the relative contributions from local modes 2 and 1, i.e. $w_{2} / w_{1}$, increase, thus breaking the initial symmetric deformation of the local mode gradually, as can be seen in Figure 16.

Compared with the long and transitional length struts, the numerical results from the variational model for the intermediate length strut shows a relatively stiffer post-buckling response when compared with the FE model. The reason is partly due to local buckling being more developed before the triggering of the secondary instability; for example, the assumed cross-section profile of the local mode, which fits well with the FE results at midspan, is not necessarily valid for the whole length. The errors would be relatively small for slender struts, since the longitudinal deflection profile is more localized. However, for the 
intermediate length strut, the longitudinal component is further distributed and the error therefore would be significantly larger.

However, it should be emphasized that the current model can capture the evolution of the longitudinal and cross-section components of the local mode well, as shown in Figures 15 and 16. It should also be noted that even though the progressive change in the local mode

(a) $q_{s}=0.001$

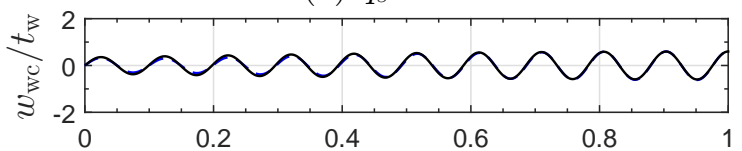

(b) $q_{s}=0.002$

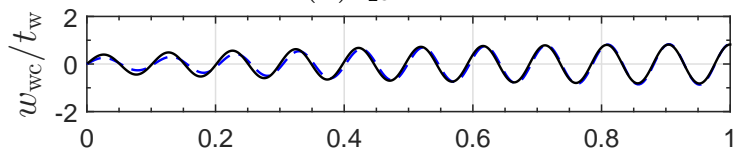

(c) $q_{s}=0.004$

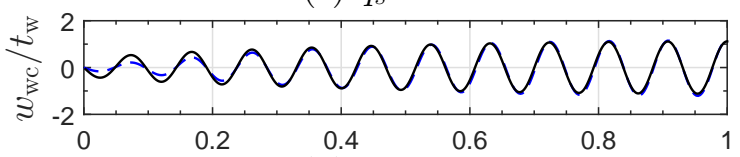

(d) $q_{s}=0.006$

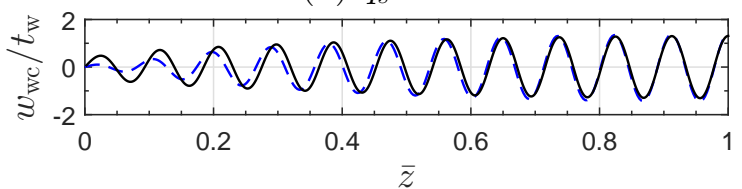

(e) $q_{s}=0.001$

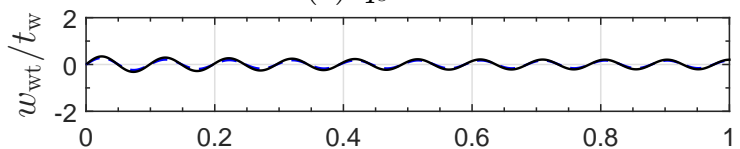

(f) $q_{s}=0.002$

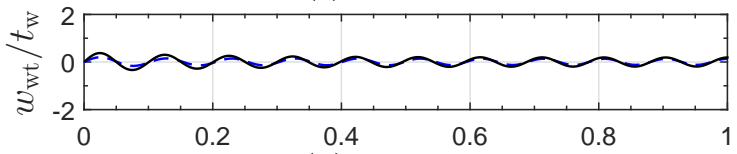

(g) $q_{s}=0.004$

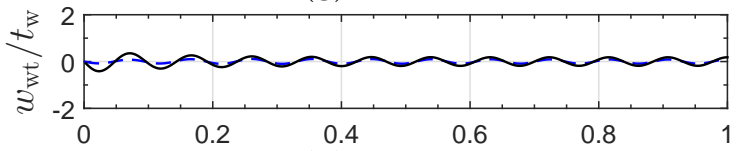

(h) $q_{s}=0.006$

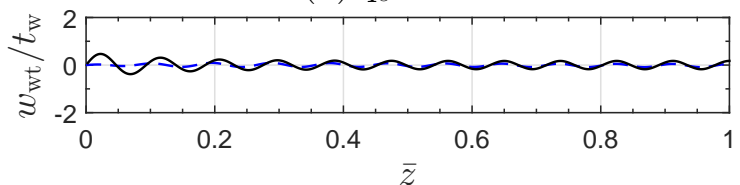

Figure 15: Evolution of the numerical solutions for $(\mathrm{a}-\mathrm{d}) w_{\mathrm{wc}} / t_{\mathrm{w}}$ and $(\mathrm{e}-\mathrm{h}) w_{\mathrm{wt}} / t_{\mathrm{w}}$ for the intermediate length strut with $L=4000 \mathrm{~mm}$. The graphs are as described in Figure 8 Note that $w_{\mathrm{wc}} / t_{\mathrm{w}}$ is now less than an order of magnitude greater than $w_{\mathrm{wt}} / t_{\mathrm{w}}$.

(a) $q_{s}=0.0005$



(b) $q_{s}=0.002$

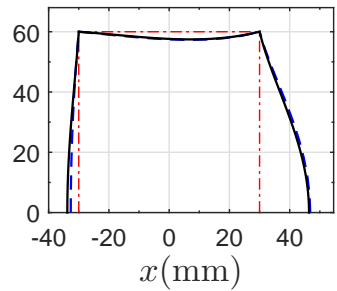

(c) $q_{s}=0.004$

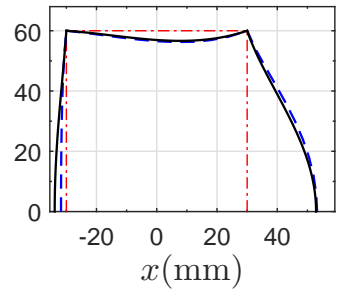

(d) $q_{s}=0.006$



Figure 16: Local deformation of the cross-section at mid-span for the intermediate length strut with $L=4000 \mathrm{~mm}$ with the increase of $q_{s}$. The graphs are as described in Figure 9

is also captured by the FE model, there are no snap-backs observed in the corresponding equilibrium paths. The variational model shows a stiffer response after the secondary bifurcation, but it predicts the critical and secondary bifurcations accurately and provides the correct trend of the post-buckling behaviour after the secondary instability. 


\subsection{Short length strut}

Previous studies [8, 29] have shown that short length struts exhibit an approximately neutral post-buckling behaviour after the secondary bifurcation point. Therefore, the main focus presently is to determine the axial stiffness reduction factor $\eta$ for the whole crosssection due to local buckling and the load at the secondary bifurcation point. The current variational model for short struts shows excellent comparisons with the FE model before the secondary bifurcation, as shown in Figure 17. However, after the secondary bifurcation, the

(a)

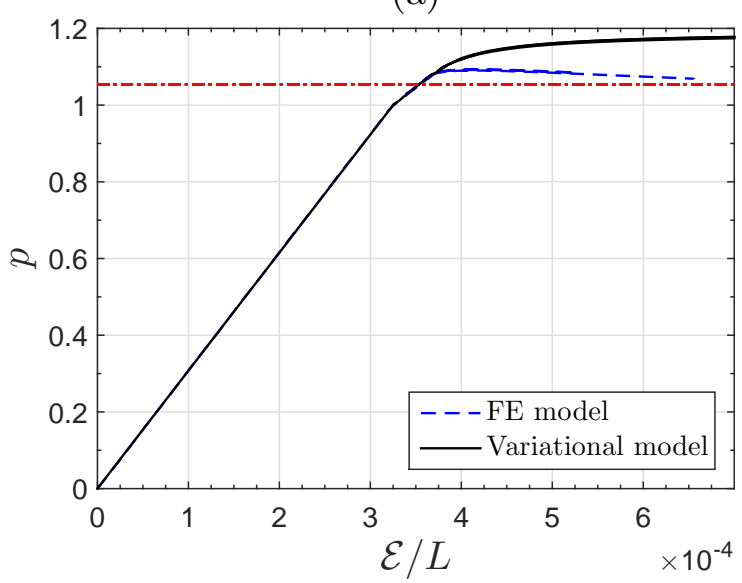

(c)

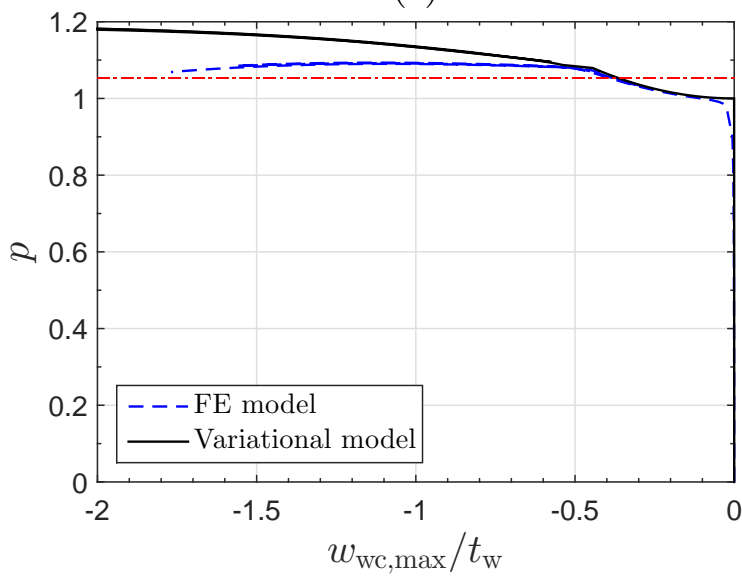

(b)

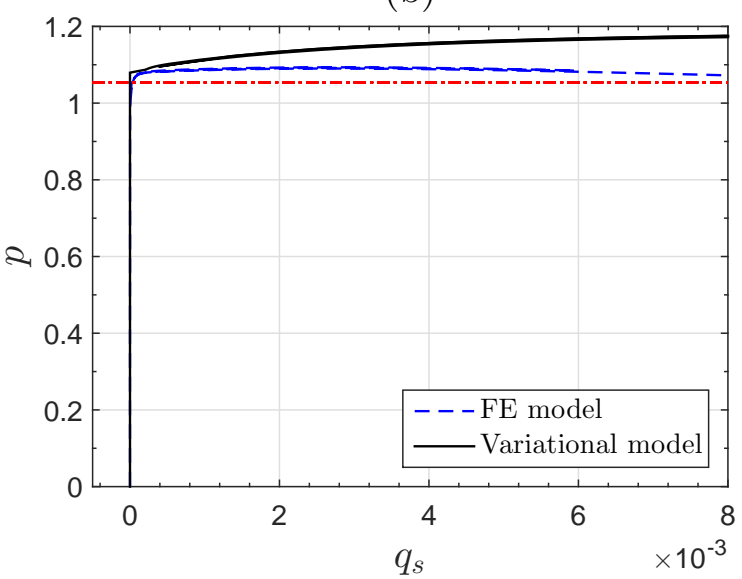

(d)

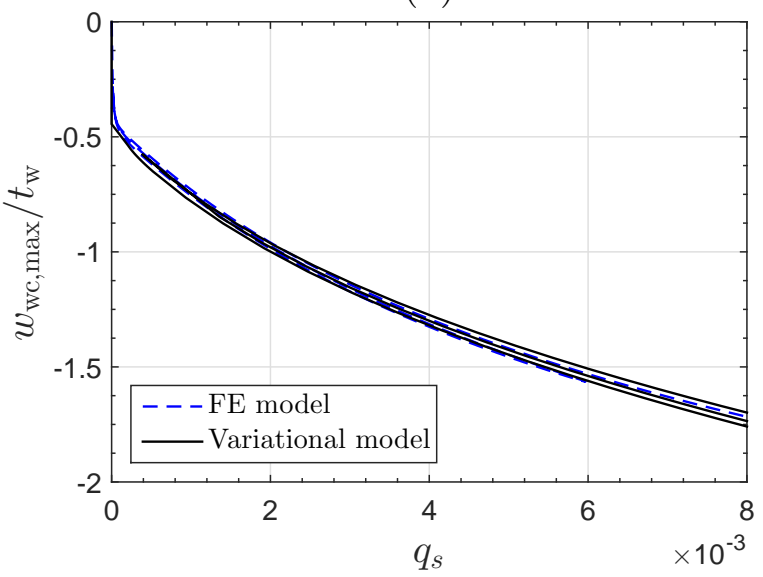

Figure 17: Nonlinear equilibrium paths for the short length strut with $L=3600 \mathrm{~mm}$ from the variational and FE models. Graphs (a-d) are as described in Figure $\mathbf{7}$ The dot-dashed line in $(\mathrm{a}-\mathrm{c})$ represents the normalized effective global buckling load $\eta P_{\mathrm{o}}^{\mathrm{C}} / P_{1}^{\mathrm{C}}=1.051$, where $\eta$ is the axial stiffness reduction factor for the whole cross-section due to the local buckling.

variational model exhibits stable post-buckling behaviour, even though there are several snap-backs. The FE model shows an approximately neutral, in fact weakly unstable, post-buckling behaviour and there are also several snap-backs observed. Although the variational model shows an overly-stiff response after the secondary bifurcation, it can 
still pinpoint the location of the secondary bifurcation point very accurately, as shown in Figure 17(d), which is meaningful for practical considerations in terms of ultimate load predictions.

It should be noted that even though the variational and FE models exhibit different post-buckling trends in the interactive buckling range, they show excellent comparisons for the local mode, i.e. the solutions for the normalized local out-of-plane displacement in the more compressed web $w_{\mathrm{wc}}$, the less compressed web $w_{\mathrm{wt}}$ (Figure 18) and the cross-section

(a) $q_{s}=0.0008$

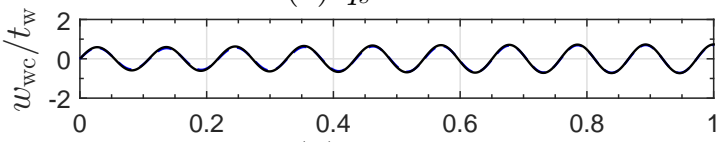

(b) $q_{s}=0.002$

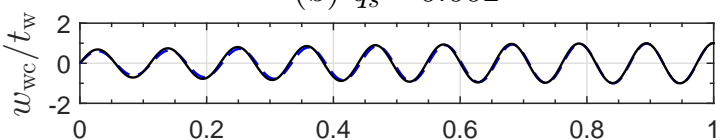

(c) $q_{s}=0.005$

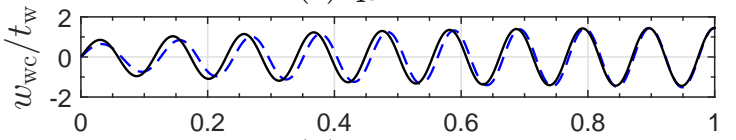

(d) $q_{s}=0.007$

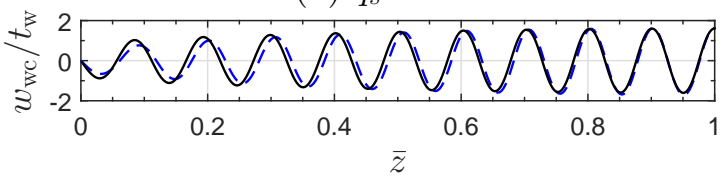

(e) $q_{s}=0.0008$

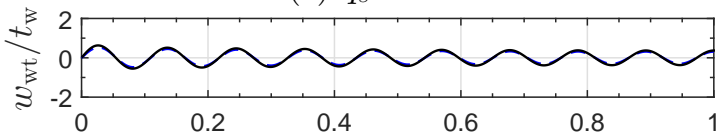

(f) $q_{s}=0.002$

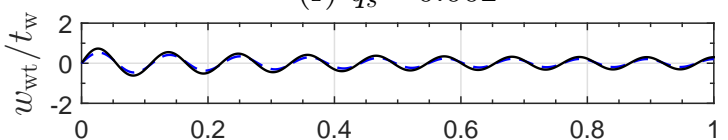

(g) $q_{s}=0.005$

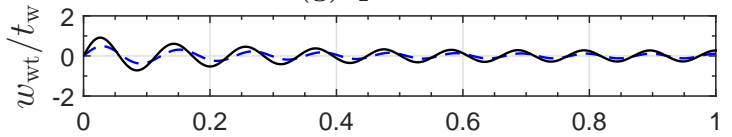

(h) $q_{s}=0.007$

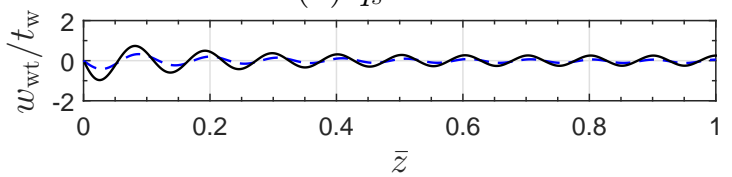

Figure 18: Evolution of the numerical solutions for $(\mathrm{a}-\mathrm{d}) w_{\mathrm{wc}} / t_{\mathrm{w}}$ and $(\mathrm{e}-\mathrm{h}) w_{\mathrm{wt}} / t_{\mathrm{w}}$ for the short length strut with $L=3600 \mathrm{~mm}$. The graphs are as described in Figure 8 Note that $w_{\mathrm{wc}} / t_{\mathrm{w}}$ is the same order of magnitude as $w_{\mathrm{wt}} / t_{\mathrm{w}}$.

deformation profile at mid span (Figure [19). In a similar fashion to the intermediate length

(a) $q_{s}=0.0008$

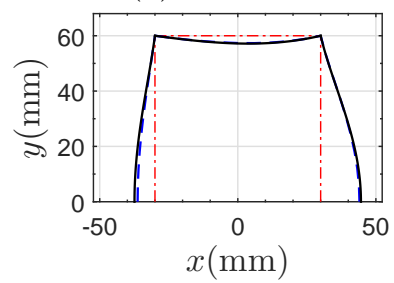

(b) $q_{s}=0.002$

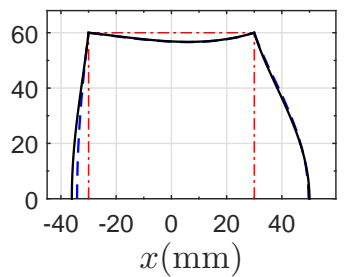

(c) $q_{s}=0.005$



(d) $q_{s}=0.007$

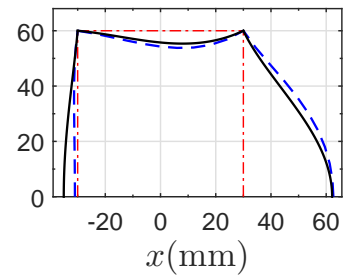

Figure 19: Local deformation of the cross-section at mid-span for the short length strut with $L=3600$ $\mathrm{mm}$ with the increase of $q_{s}$. The graphs are as described in Figure 9

strut, the ratio $w_{2} / w_{1}$ increases alongside the increase of $q_{s}$, leading to the transition of $w_{\mathrm{wc}}$ from being initially distributed to localized and the decay of the $w_{\mathrm{wt}}$ amplitude. Moreover, a change in the number of peaks and troughs is also observed, which corresponds to the snapback in the equilibrium path, as shown in Figure 17. As for the cross-section deformation 
at mid-span, a profile transition from that dominated by mode 1 to one dominated by mode 2 is also observed.

Compared with the other example struts, the strain level is much higher at and beyond the secondary bifurcation point, which makes the assumptions that are applicable to slender struts less valid. All the reasons leading to the stiffer post-buckling response in the transitional and intermediate length struts can also be used to explain the overly-stiff response of the strut predicted by the variational model. Moreover, it should be recalled that any explicitly transverse displacements within the cross-section are omitted in the current variational model and only Poisson's ratio effects are accounted in those directions. However, the corner joints of the cross-section would move with the progress of mode interaction, even though the magnitude is quite small, as illustrated in Figure 20, The effects

(a) Before buckling

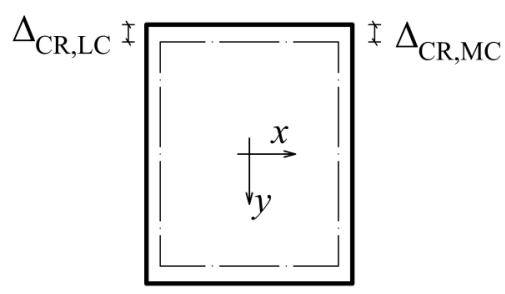

(b) Pure local buckling

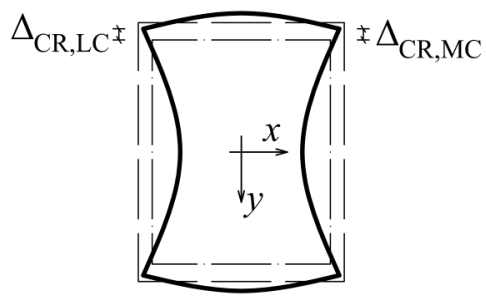

(c) Interactive buckling

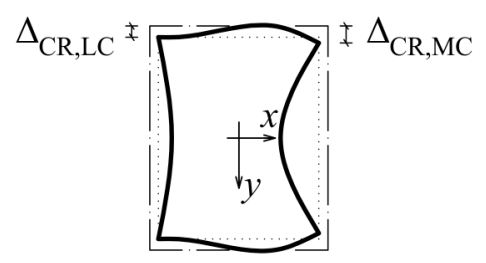

Figure 20: In-plane displacements within the cross-section. (a) Expansion due to the Poisson's ratio effect before local buckling occurs. (b) Symmetrical contraction due to pure local buckling. (c) Asymmetrical contraction due to interactive buckling. The thick solid line and the dot-dashed line represent the deformed and undeformed shape respectively. The dashed line in (b) represents the deformed shape before buckling. Also shown are the vertical displacements of the more and less compressed web-flange joints $\Delta_{\mathrm{CR}, \mathrm{MC}}$ and $\Delta_{\mathrm{CR}, \mathrm{LC}}$. The small reduction in the overall cross-section depth and width would account for some reduction in the post-buckling stiffness.

become more significant in the short length struts, as shown in Figure 21, which presents results from the FE model. The omission of the explicitly transverse displacements has also been discussed recently in a related study [67]. To capture the full post-buckling path accurately, a local mode description that includes the cross-section in-plane displacement field, i.e. the transverse displacements in both flanges and webs, would be required [67].

From the FE results and previous studies [27], it has been shown that the strut is neutrally stable after the secondary bifurcation. Therefore, locating the secondary bifurcation point is in fact sufficient for practical strength prediction. Moreover, since the variational model shows excellent agreement with the FE model before the secondary bifurcation, the stiffness reduction factor $\eta$ can be obtained from the load-end-shortening relationship with very good accuracy:

$$
\eta=\frac{(\mathrm{d} P / \mathrm{d} \mathcal{E})_{\text {post }}}{(\mathrm{d} P / \mathrm{d} \mathcal{E})_{\text {pre }}}=0.645 .
$$

Therefore, the Euler buckling load for the locally buckled strut $\eta P_{\mathrm{o}}^{\mathrm{C}}$ can be calculated and is plotted with the dot-dashed line in Figure 17. In comparison with the ultimate load from 
(a)

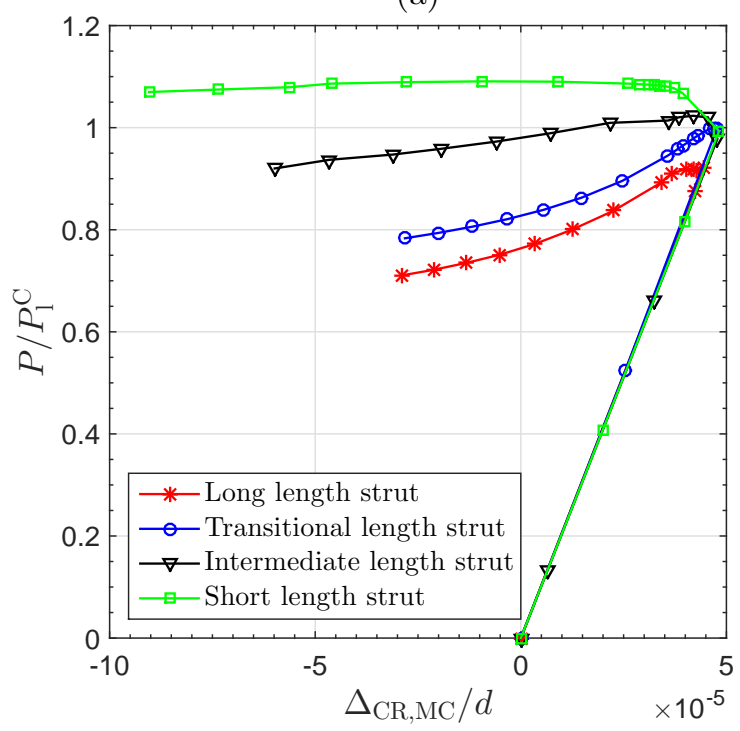

(b)

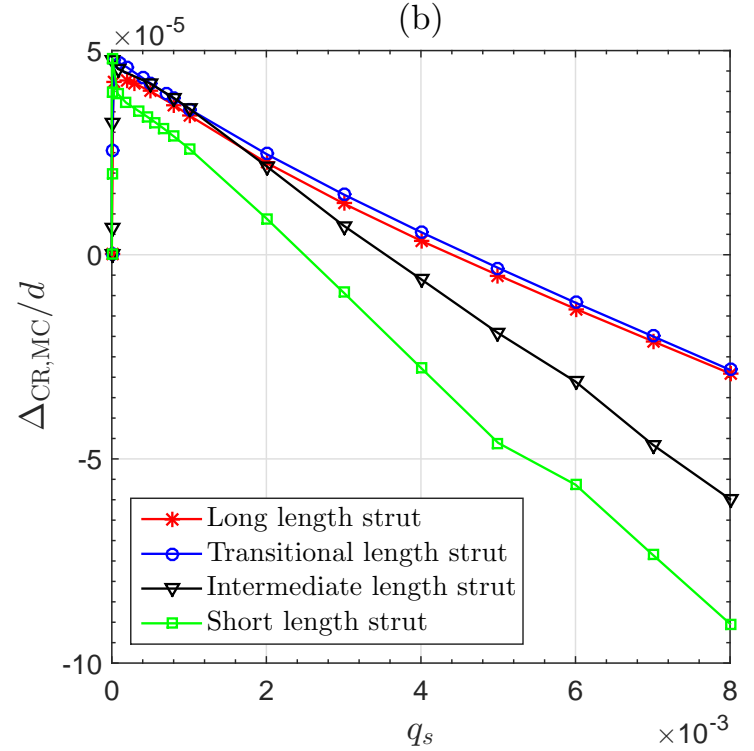

Figure 21: The normalized vertical in-plane displacement in the more compressed web $\Delta_{\mathrm{CR}, \mathrm{MC}} / d$ at midspan (see Figure 20) versus (a) the normalized load $P / P_{1}^{\mathrm{C}}$ and (b) $q_{s}$ for struts with different lengths from the FE results. The variational model currently omits this displacement.

the FE model, $P_{\mathrm{u}, \mathrm{FE}}$, the variational model provides a safe, yet accurate strength prediction where $\eta P_{\mathrm{o}}^{\mathrm{C}} / P_{\mathrm{u}, \mathrm{FE}}=0.976$. Hence, it may be concluded that the developed variational model has been validated for all the zones considered and may now be investigated further.

\section{Discussion and comparison with van der Neut's model}

A parametric study is now presented to determine the respective boundaries for the four distinct length domains using the developed variational model. The numerical results are presented in Figure 22 in the style of the classical van der Neut curve that was shown in Figure 1(b). The shape of the curve is very similar to previous similar results for stiffened panels [29, 26] and I-section struts [27, 44]. The ultimate load for perfect long and transitional length struts is the corresponding critical buckling load $P^{\mathrm{C}}$. The triggering of mode interaction leads to unstable post-buckling behaviour. Particularly for transitional length struts (zone 2), since the critical and secondary bifurcations are practically coincident, reaching the ultimate load in practice may not be possible due to the inherently high degree of sensitivity to imperfections that would be expected [6, 16, 17, 18, 19, 26, 25, 28]. For intermediate length struts (zone 3), stable post-buckling behaviour is exhibited after the critical bifurcation, as shown in Figure 14. Therefore, the ultimate load is higher than the critical (local) buckling load. For the ultimate load prediction, the variational model shows excellent agreement with the FE model, as shown in Figure 22. For the short length struts (zone 4), stable post-buckling behaviour is also exhibited after the critical bifurcation and approximately neutrally stable post-buckling behaviour after the secondary bifurcation. The variational model can also predict the ultimate load with good accuracy 


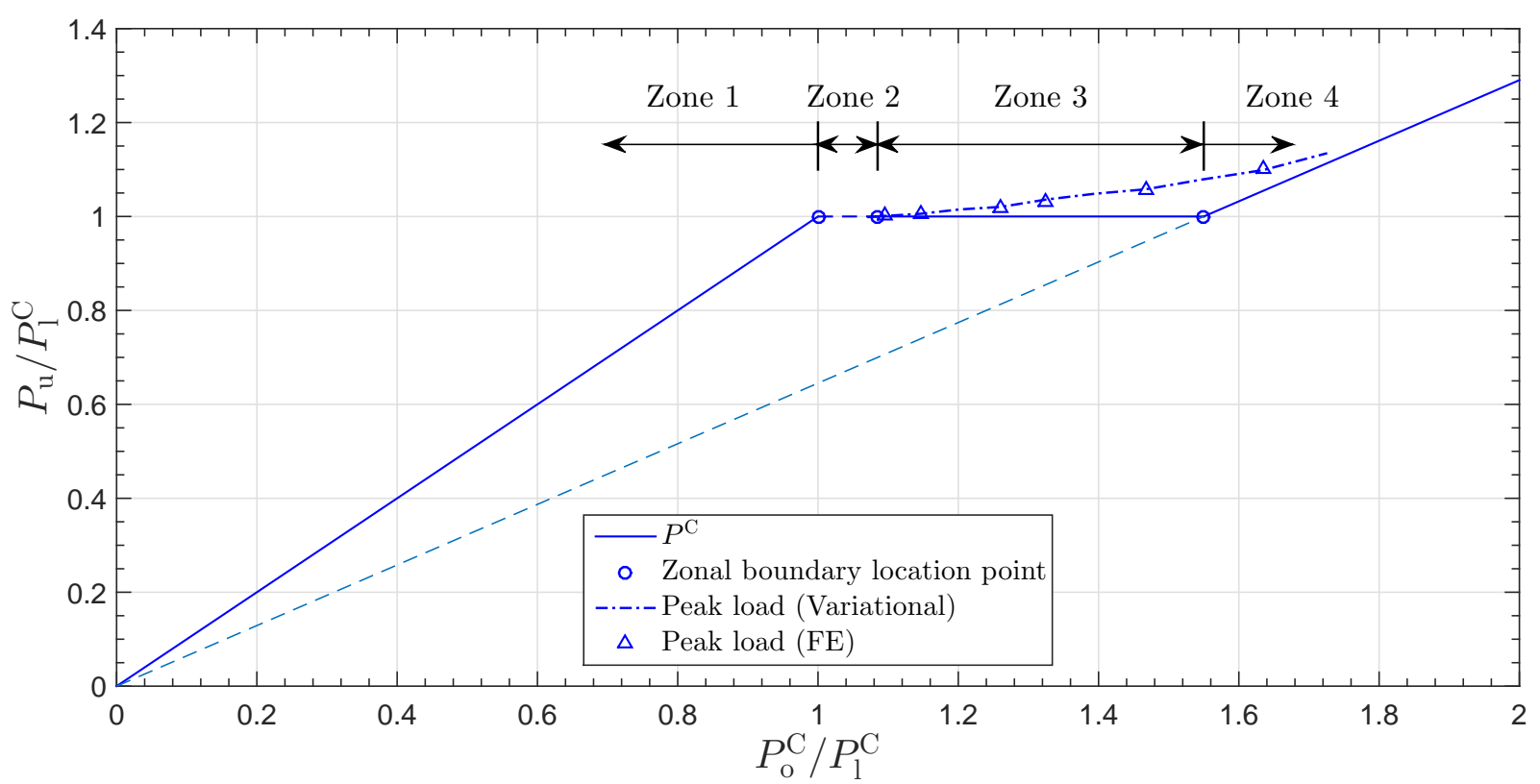

Figure 22: The numerically obtained van der Neut curve for struts with cross-section and material properties presented in Table 1 It should be noted that the slope of dashed line is in fact equal to the axial stiffness reduction factor for the whole cross-section due to pure local buckling.

and $\eta P_{\mathrm{o}}^{\mathrm{C}}$ provides a safe, yet relatively accurate, prediction for the ultimate load.

Since the struts exhibit distinct behaviour in different zones, it is beneficial to determine the boundary between each zone for practical engineering purposes. The boundary between zones 1 and 2 is very straightforward since this is by definition where the global buckling load $P_{\mathrm{o}}^{\mathrm{C}}$ and the local buckling load $P_{1}^{\mathrm{C}}$ precisely coincide.

The boundary between zones 2 and 3 corresponds to the competing mode between pure local and global buckling due to the cross-section bending stiffness reduction caused by local buckling of the compressed side of the cross-section, as shown in Figure 23. The buckling loads for the depicted modes (I) and (II) are the local buckling load $P_{1}^{\mathrm{C}}$ and the reduced global buckling load $\Phi_{\mathrm{r}} P_{\mathrm{o}}^{\mathrm{C}}$ respectively, where $\Phi_{\mathrm{r}}$ is the bending stiffness reduction factor due to local buckling of the more compressed web and flanges. At the zonal boundary, the ultimate load for the two competing modes would be the same, i.e. $\Phi_{\mathrm{r}} P_{\mathrm{o}}^{\mathrm{C}}=P_{1}^{\mathrm{C}}$, which leads to the value $P_{\mathrm{o}}^{\mathrm{C}} / P_{1}^{\mathrm{C}}=1 / \Phi_{\mathrm{r}}$.

For the ideal two-flange model originally presented by van der Neut, $\Phi_{\mathrm{r}}$ can be obtained from Engesser's so-called 'double modulus' theory [4]. The current variational formulation cannot in fact replicate the results of van der Neut's idealization precisely since shear strain is neglected in the latter. However, a simplified rectangular hollow section strut model with pinned corners within the cross-section, which is very similar to van der Neut's ideal model, may be used for validation and illustration purposes, as shown in Figure 23(b). Since each corner within the cross-section is pinned, the web and flange plates buckle independently. In zone 2, when global buckling is triggered, only the more compressed web remains buckled, as shown in Figure 23(c), and the corresponding effective cross-section stiffness distribution 
(a)

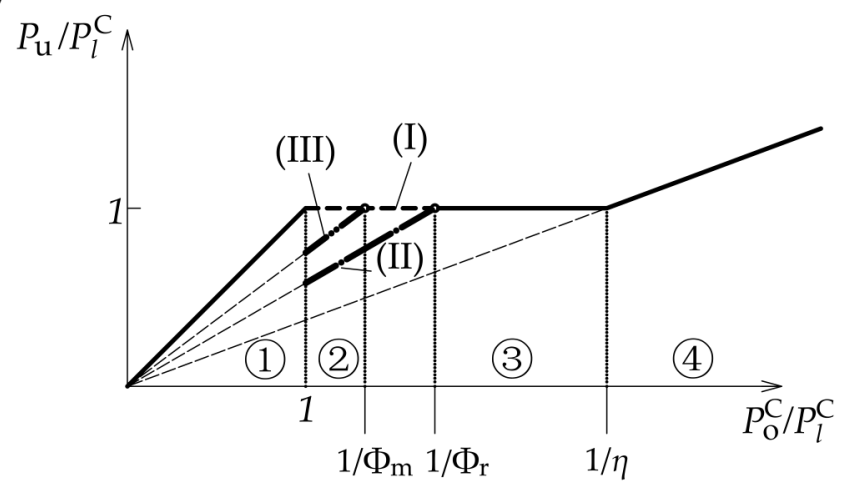

(b)

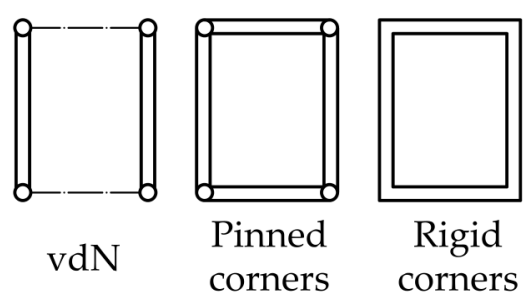

(c)

Plan view:

(I)

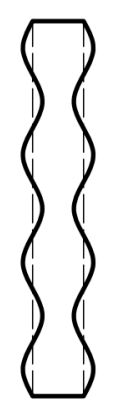

(II)

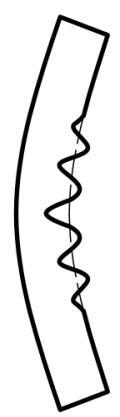

\section{Cross-section:}

(III)

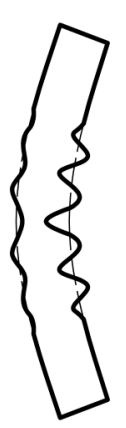

(I)
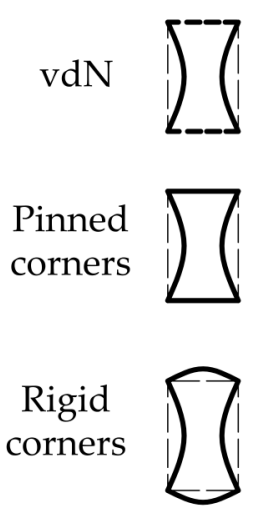

(II)

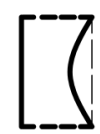

N/A

(III)

N/A


Figure 23: (a) The van der Neut-type curve sketched with the competing buckling modes in zone 2. (b) Cross-section flange--web conditions for van der Neut's model (vdN), a rectangular hollow section with pinned corners and a rectangular hollow section with rigid corners. (c) Competing buckling modes in zone 2 for three different models: (I) pure local buckling; (II) global buckling due to the cross-section bending stiffness reduction caused by local buckling in the compressed web and flanges; (III) transitional state between (I) and (II). The bending stiffness reduction factor due to local buckling in the more compressed web and flanges is $\Phi_{\mathrm{r}}$ and the equivalent reduction factor for the transitional state (III) is $\Phi_{\mathrm{m}}$. 
is shown in Figure 24(a). Based on the cross-section stiffness distribution, using the double modulus theory, the details of which are in the Appendix, by setting $\eta_{\mathrm{f}}(x)$ in Eqs. (A.2) and (A.8) to unity, the following expression for $\Phi_{\mathrm{r}}$ is obtained:

$$
\Phi_{\mathrm{r}}=\frac{\left[\left(\bar{\eta}_{\mathrm{w}}+1\right)^{2} t_{\mathrm{w}}^{2}+12 b^{2} \bar{\eta}_{\mathrm{w}}\right] \phi_{\mathrm{c}}^{2}+2\left[\left(\bar{\eta}_{\mathrm{w}}+1\right) t_{\mathrm{w}}^{2}+4\left(\bar{\eta}_{\mathrm{w}}+1\right) b^{2}\right] \phi_{\mathrm{c}} \phi_{\mathrm{t}}+4 b^{2} \phi_{\mathrm{t}}^{2}}{2\left[2 \phi_{\mathrm{t}}+\left(1+\bar{\eta}_{\mathrm{w}}\right) \phi_{\mathrm{c}}\right]\left[\left(3 b^{2}+t_{\mathrm{w}}^{2}\right) \phi_{\mathrm{c}}+b^{2} \phi_{\mathrm{t}}\right]} .
$$

For the strut with the same cross-section and material properties as the example struts presented in Table 1. $\Phi_{\mathrm{r}}=1 / 1.462$, which is very close to the value computed using the variational model, where $P_{\mathrm{o}}^{\mathrm{C}} / P_{1}^{\mathrm{C}}=1.461$. It validates the effectiveness of the double modulus theory for predicting the boundary between zones 2 and 3 for such cases. Moreover, it should also be noted that the value is much smaller than that obtained by van der Neut, where $\bar{\eta}_{\mathrm{w}}=0.408$ and $\Phi_{\mathrm{r}}=2 \bar{\eta}_{\mathrm{w}} /\left(1+\bar{\eta}_{\mathrm{w}}\right)=1 / 1.725$, even though the equivalent axial stiffness reduction factor $\bar{\eta}_{\mathrm{w}}$ in the more compressed web is adopted as the same value due to the pinned corners within the cross-section. The difference arises due to the inclusion of both flanges, which contribute the full elastic modulus $E$.

For the hollow section with rigid flange--web joints, which is the most widely applicable for engineering practice, the axial stiffness distribution in the cross-section for the competing mode is not as straightforward as the pinned corner case due to the interaction of the flanges and webs within the cross-section. Therefore, three different stiffness distribution candidate schemes are considered, as shown in Figure 24, and the corresponding

(a) Scheme 1

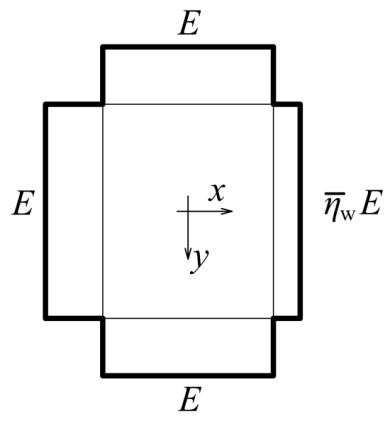

(b) Scheme 2



(c) Scheme 3

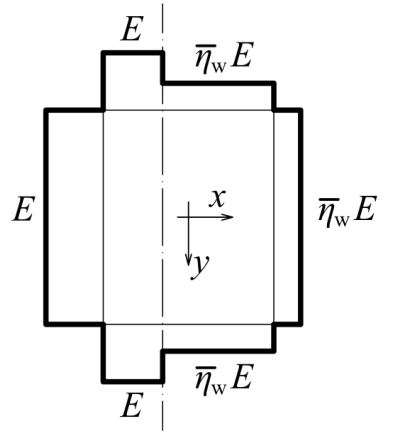

Figure 24: Cross-section axial stiffness distribution schemes, where the stiffness is directly proportional to the material Young's modulus $E$ and $\bar{\eta}_{\mathrm{w}}$ is the equivalent axial stiffness reduction factor due to local buckling of the more compressed web.

relationship between $1 / \Phi_{\mathrm{r}}$ and $\bar{\eta}_{\mathrm{w}}$ can be established using Eq. A.8). Figure 25 shows the relationship for the strut with the same cross-section and material properties as the example struts (see Table 1). The value of $1 / \Phi_{\mathrm{r}}$, i.e. $P_{\mathrm{o}}^{\mathrm{C}} / P_{1}^{\mathrm{C}}$ at the boundary between zones 2 and 3 , increases with the reduction of the effective axial stiffness of the whole section from schemes 1 to 3 as well as with the reduction of the equivalent axial stiffness of the more compressed web $\bar{\eta}_{\mathrm{w}}$. Since the actual effective axial stiffness of the more compressed side of the flanges for the competing mode would be reduced due to local buckling, as shown 


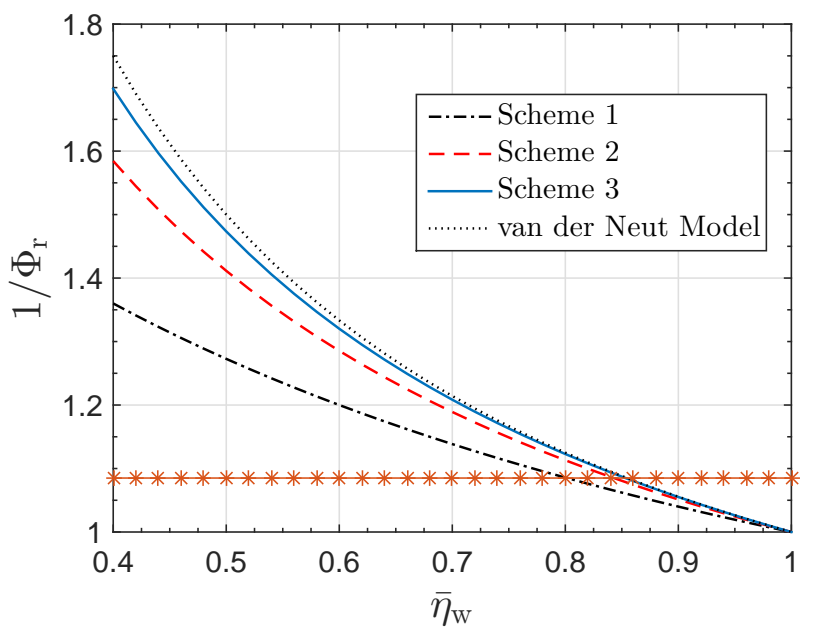

Figure 25: The relationship between $1 / \Phi_{\mathrm{r}}$ and $\bar{\eta}_{\mathrm{w}}$ for the cross-section stiffness distributions presented in Figure 24 and the expression proposed by van der Neut. The asterisk line represents the equivalent $1 / \Phi_{\mathrm{r}}$ for the example struts obtained from the current variational model results that are validated by FE.

in Figures 26 and A.1(b), scheme 1 would provide the lowest limiting prediction for $1 / \Phi_{\mathrm{r}}$ if the double modulus approach were to be applicable for the cross-section case with rigid flange-web joints.

However, the parametric study (see Figure 22) shows that the value of $P_{\mathrm{o}}^{\mathrm{C}} / P_{1}^{\mathrm{C}}$ at the boundary of zones 2 and 3 for the example struts with rigid joints between flanges and webs is 1.085, which corresponds to the asterisk line in Figure 25. Since it has been noted that scheme 1 provides the lowest limiting prediction of $1 / \Phi_{\mathrm{r}}$, the corresponding value of $\bar{\eta}_{\mathrm{w}}$ for the more compressed web should be even larger than 0.8, as shown in Figure 25. Even though the rigid flange-web joints can provide more restraint to the more compressed web, thus leading to a higher post-buckling stiffness, the value of $\bar{\eta}_{\mathrm{w}}$ for the more compressed web using the isolated more compressed web model [52] is 0.46 . Therefore, it seems that including the contribution of the flanges and the rigid flange-web joints not only introduces additional stiffness when compared to the pinned cross-section case but also potentially more competing modes, which diminishes the validity of the double modulus approach for such cases.

It should be emphasized that in both the van der Neut model and the case where the cross-section has pinned corners, the effective axial stiffness in the more compressed web remains approximately the same before and after the transition from the pure local buckling mode to local-global interactive buckling due to the simply-supported boundary condition of the unloaded edges, as shown in Figure 23(c). However, for the rigid flangeweb joint case, there is a reduction in the deformation of the more compressed side after the transition, which is particularly apparent in the flanges, as can be seen from Figure 26. It suggests that the unloading of the less compressed web and flanges affects the stiffness distribution in the more compressed side of the cross-section, which does not occur in van der Neut's ideal model and the case of the cross-section with pinned corners. It implies 




Figure 26: Cross-section deformation for the pure local buckling case (dot-dashed line) using Eqs. (4) and (5) and the global buckling induced local buckling case (solid line) using Eqs. (11)-(13).

that there is an increase in the axial stiffness of the more compressed side of the crosssection and it can thus resist higher compressive stresses. The condition for the boundary between zones 2 and 3 can thus be written as $P_{1}^{\mathrm{C}}=\Phi_{\mathrm{r}} P_{\mathrm{o}}^{\mathrm{C}}+\delta P$, where $\delta P$ is the load increase due to the stiffness redistribution in the cross-section caused by the less compressed side of the cross-section. Therefore, there should be an additional transitional stage (III) between these two more distinct states, as shown in Figure 23. This is also confirmed by the numerical results of the cross-section deformation shape for the transitional length strut. The existence of the transitional stage moves the boundary of zones 2 and 3 to the left when compared with stage (II), as shown in Figure 23(a), where $P_{\mathrm{o}}^{\mathrm{C}} / P_{1}^{\mathrm{C}}$ is equal to $\left(1-\delta P / P_{1}^{\mathrm{C}}\right) / \Phi_{\mathrm{r}}$. This finding is also in accord with results from previous work on stiffened panels [29, 26] and I-section struts [27, 44]. Since the unstable range is notorious for its imperfection sensitivity [25, 26], a somewhat reduced imperfection sensitivity range may be expected for such struts although further studies are required for this to be confirmed and quantified.

The boundary between the intermediate length struts (zone 3) and the short length struts (zone 4) is the same as that in van der Neut's curve, where $P_{\mathrm{o}}^{\mathrm{C}} / P_{1}^{\mathrm{C}}=1 / \eta=1.550$. From Figure 17 $(\mathrm{a}-\mathrm{c})$, with the decrease of the strut length, the peak load would converge to $\eta P_{\mathrm{o}}^{\mathrm{C}}$, as shown in Figure 22. However, it should be noted that the straight line in zone 4 would in fact be curved with a decreasing slope due to the decreasing stiffness reduction factor caused by the effects of large deflections in plates; a similar effect would occur if a yield stress were introduced into the material model [44].

Finally, it should be noted that the factors listed for the movement of the boundary between zones 2 and 3 also affect the value of $P_{\mathrm{o}}^{\mathrm{C}} / P_{1}^{\mathrm{C}}$ at the boundary of zones 3 and 4. However, since the local buckling mode is more developed before mode interaction is triggered, the effects of stiffness redistribution would be much smaller. Moreover, adopting $\eta$ as the bending stiffness reduction factor would provide an accurate, yet safe ultimate 
load prediction.

\section{Concluding remarks}

A nonlinear variational model describing the interactive buckling of elastic thin-walled rectangular hollow section struts with different lengths under pure compression has been developed using variational principles. Numerical examples, considering four representative lengths corresponding to distinct parts of the van der Neut curve, have been presented and validated using the commercial FE package ABAQUS. Using the validated variational model, the boundaries of four distinct length-dependent domains have been identified for realistic cross-section characteristics for the first time.

Unstable post-buckling behaviour due to mode interaction was observed in long, transitional and intermediate length struts. Potentially dangerous behaviour, i.e. a sharp drop in the load capacity immediately after the ultimate load is reached, has been identified where the global buckling load is close to the local buckling load. A progressive change in the local buckling mode is observed within the nonlinear post-buckling range in all struts. In particular, for intermediate and short length struts, snap-backs that correspond to the change in the number of troughs and peaks in the local mode that are akin to cellular buckling, are highlighted. The parametric study on strut length shows that the notoriously unstable post-buckling range for rectangular hollow-section struts is in fact significantly smaller than that predicted by van der Neut's idealized model and the stiffness reduction factor is also considerably higher. The reason for this difference has been identified as being derived from the interaction between the individual plates due to the rigid corners within the cross-section, which lead to stiffness redistribution. This is in contrast with the assumption that the corners are pinned where the web and flange plates behave independently.

Work is currently being conducted to extend the model to quantify the effects of initial geometric imperfections. Moreover, parametric studies on the geometric properties, i.e. varying the global and local slendernesses, will be conducted to produce relationships that include the effects of geometric imperfections, thus providing guidance to engineers for designing thin-walled rectangular hollow section struts with geometries that are susceptible to modal interactions.

\section{Acknowledgements}

Financial support for Jiajia Shen was provided by the President's PhD Scholarship scheme from Imperial College London. The authors are also grateful to Dr Adam J. Sadowski from Imperial for his advice in developing the finite element model within ABAQUs.

\section{Appendix A. Zone 2-3 boundary determination from double modulus theory}

Van der Neut predicted the boundary between zones 2 and 3 using Engesser's 'doublemodulus' theory [4]. The same approach is adopted currently to predict the boundary for 
the rectangular hollow section strut. It has been verified that global and local buckling is triggered simultaneously in zone 2. Prior to buckling $(w=0)$, the stress distribution is uniform, $\sigma_{0}=P / A$. It has been assumed in the current work that plane cross-sections remain plane but not normal to the deflected centre line of the strut at the initial buckling stage. At the commencement of mode interaction, the compressed side of the strut would remain buckled and undergo further shortening, hence further loading, and the less compressed side would undergo relative extension, hence unloading. Somewhere within the cross-section, there is a neutral axis at which the axial strain would not change; its distance from the more and less compressed sides of the strut being denoted as $b_{1}$ and $b_{2}$ respectively, where $b_{1}+b_{2}=b$, as shown in Figure A.1(b). The effective axial stiffness in

(a)

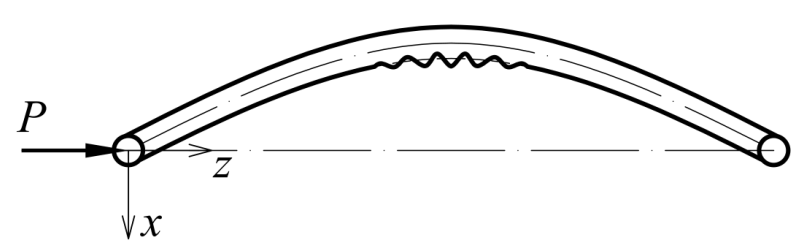

(b)

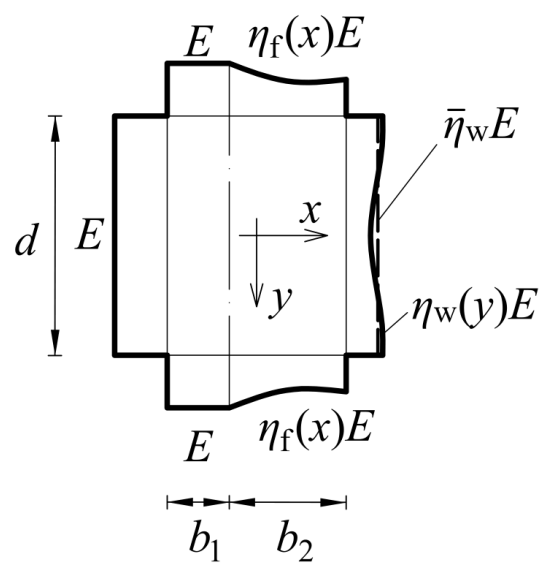

(c)

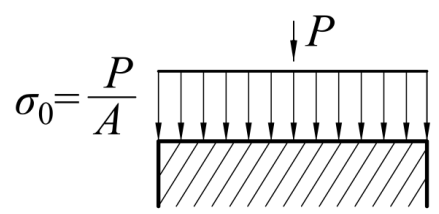

(d)

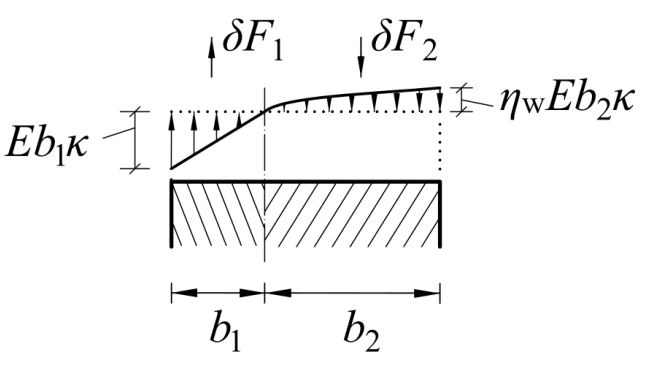

Figure A.1: (a) Interactive buckling in a simply-supported rectangular hollow section strut under the concentric axial load $P$. (b) Cross-section stiffness distribution at the commencement of interactive buckling. The functions $\eta_{\mathrm{f}}(x)$ and $\eta_{\mathrm{w}}(y)$ describe the stiffness reduction distributions for the flanges and the more compressed web respectively due to local buckling. (c) Normal stress distribution before mode interaction is triggered. (d) Variation in the normal stress due to mode interaction. The curvature due to global buckling is $\kappa ; \delta F_{1}$ and $\delta F_{2}$ are the relative changes in loading in the less and more compressed sides of the cross-section respectively, which include the unloading and loading of the flanges and webs.

the more compressed side of the cross-section would drop due to plate buckling. It is assumed that the distribution of the effective elastic axial stiffness is proportional to $\eta_{\mathrm{f}}(x) E$ and $\eta_{\mathrm{w}}(y) E$ in the flanges and the more compressed web respectively.

According to the assumption of plane cross-sections remaining plane and the assumed effective axial stiffness distribution above, the incremental resultant force within the less 
compressed side $\delta F_{1}$ and the more compressed side $\delta F_{2}$ can be obtained, thus:

$$
\begin{aligned}
& \delta F_{1}=\int_{-d / 2}^{d / 2} E t_{\mathrm{w}} \varepsilon_{\mathrm{wt}} \mathrm{d} y+2 \int_{0}^{b_{1}} E t_{\mathrm{f}} \varepsilon_{\mathrm{f}} \mathrm{d} x=E b_{1} \kappa\left(d t_{\mathrm{w}}+b_{1} t_{\mathrm{f}}\right), \\
& \delta F_{2}=\int_{-d / 2}^{d / 2} \eta_{\mathrm{w}}(y) E \varepsilon_{\mathrm{wc}} \mathrm{d} y+2 \int_{0}^{b_{2}} \eta_{\mathrm{f}}(x) E t_{\mathrm{f}} \varepsilon_{\mathrm{f}} \mathrm{d} x=E b_{2} \kappa\left(\bar{\eta}_{\mathrm{w}} d t_{\mathrm{w}}+\frac{t_{\mathrm{f}}}{b_{2}} \int_{0}^{b_{2}} x \eta_{\mathrm{f}}(x) \mathrm{d} x\right),
\end{aligned}
$$

where $\varepsilon_{\mathrm{wt}}=b_{1} \kappa$ and $\varepsilon_{\mathrm{wc}}=b_{2} \kappa$ are the direct strains in the less compressed and more compressed webs respectively; $\varepsilon_{\mathrm{f}}=x \kappa$ is the direct strain in the flanges; $\kappa$ is the curvature due to global buckling; $\bar{\eta}_{\mathrm{w}}=\int_{-d / 2}^{d / 2} \eta_{\mathrm{w}}(y) \mathrm{d} y$ is the equivalent stiffness reduction factor in the more compressed web.

The condition of constant axial load requires that the resultants $\delta F_{1}$ and $\delta F_{2}$ of the incremental normal stress in the more and less compressed sides must be of equal magnitudes, thus:

$$
\delta F_{1}=\delta F_{2} .
$$

As for the moment $M$ in the cross-section,

$$
M=M_{1}+M_{2},
$$

where:

$$
\begin{aligned}
M_{1} & =\int_{b_{1}-t_{\mathrm{w}} / 2}^{b_{1}+t_{\mathrm{w}} / 2} \int_{-d / 2}^{d / 2} x E t_{\mathrm{w}} \varepsilon_{\mathrm{wt}} \mathrm{d} y \mathrm{~d} x+2 \int_{0}^{b_{1}} x E t_{\mathrm{f}} \varepsilon_{\mathrm{f}} \mathrm{d} x \\
& =E \kappa\left(b_{1}^{2} d t_{\mathrm{w}}+\frac{d t_{\mathrm{w}}^{3}}{12}+\frac{2 b_{1}^{3} t_{\mathrm{f}}}{3}\right) \\
M_{2} & =\int_{b_{2}-t_{\mathrm{w}} / 2}^{b_{2}+t_{\mathrm{w}} / 2} \int_{-d / 2}^{d / 2} x \eta_{\mathrm{w}}(y) E t_{\mathrm{w}} \varepsilon_{\mathrm{wc}} \mathrm{d} y \mathrm{~d} x+2 \int_{0}^{b_{2}} x \eta_{\mathrm{f}}(x) E t_{\mathrm{f}} \varepsilon_{\mathrm{f}} \mathrm{d} x \\
& =E \kappa\left[\bar{\eta}_{\mathrm{w}}\left(b_{2}^{2} d t_{\mathrm{w}}+\frac{d t_{\mathrm{w}}^{3}}{12}\right)+2 \int_{0}^{b_{2}} x^{2} \eta_{\mathrm{f}}(x) t_{\mathrm{f}} \mathrm{d} x\right],
\end{aligned}
$$

which can be rewritten as:

$$
M=\Phi_{\mathrm{r}} E I \kappa,
$$

with $I=b^{3} t_{\mathrm{f}} / 6+b^{2} d t_{\mathrm{w}}+d t_{\mathrm{w}}^{3} / 6$ being the second moment of area about the weak neutral axis of the entire cross-section before buckling. Therefore, the bending stiffness reduction factor due to local buckling in the more compressed side can be written as:

$$
\begin{aligned}
\Phi_{\mathrm{r}} & =\frac{\left(M_{1}+M_{2}\right)}{E I \kappa} \\
& =\frac{\left(b_{1}^{2} d t_{\mathrm{w}}+d t_{\mathrm{w}}^{3} / 12+2 b_{1}^{3} t_{\mathrm{f}} / 3\right)+\bar{\eta}_{\mathrm{w}}\left(b_{2}^{2} d t_{\mathrm{w}}+d t_{\mathrm{w}}^{3} / 12\right)+2 \int_{0}^{b_{2}} x^{2} \eta_{\mathrm{f}}(x) t_{\mathrm{f}} \mathrm{d} x}{b^{3} t_{\mathrm{f}} / 6+b^{2} d t_{\mathrm{w}}+d t_{\mathrm{w}}^{3} / 6},
\end{aligned}
$$

where $b_{1}$ and $b_{2}$ can be obtained explicitly using the constant axial force condition from Eq. (A.3). It should be noted that if $t_{\mathrm{f}} \rightarrow 0$ and $b \gg t_{\mathrm{w}}$, i.e. where both flanges have a negligible thickness, $\Phi_{\mathrm{r}}$ would reduce to the expression developed in []], where $\Phi_{\mathrm{r}}=2 \bar{\eta}_{\mathrm{w}} /\left(1+\bar{\eta}_{\mathrm{w}}\right)$. 


\section{References}

[1] F. Bleich, Buckling strength of metal structures, McGraw-Hill, New York, 1952.

[2] S. P. Timoshenko, J. M. Gere, Theory of elastic stability, McGraw-Hill, New York, 1961.

[3] B. Budiansky, J. W. Hutchinson, Buckling-Progress and challenge, in: J. F. Besseling, A. M. A. van der Heijden (Eds.), Trends in solid mechanics: Dedicated to the 65th birthday of W. T. Koiter, Delft University Press, Delft, the Netherlands, 1979, pp. 93-116.

[4] Z. P. Bažant, L. Cedolin, Stability of structures: Elastic, inelastic, fracture, and damage theories, Oxford University Press, Oxford, 1991.

[5] W. T. Koiter, On the stability of elastic equilibrium, Ph.D. thesis, Technische Hogeschool, Delft (Technological University of Delft), (English translation issued as NASA, Tech. Trans., F 10, 833, 1967) (1945).

[6] J. M. T. Thompson, G. W. Hunt, A general theory of elastic stability, Wiley, London, 1973.

[7] S. Cherry, The stability of beams with buckled compression flanges, Struct. Eng. 38 (9) (1960) 277-285.

[8] A. van der Neut, The interaction of local buckling and column failure of thin-walled compression members, in: Proceedings of the 12th International Congress on Applied Mechanics, Springer, 1969, pp. 389-399.

[9] J. M. T. Thompson, G. W. Hunt, Elastic instability phenomena, Wiley, London, 1984.

[10] V. Gioncu, General theory of coupled instabilities, Thin-Walled Struct. 19 (2) (1994) 81-127.

[11] J. Becque, K. J. R. Rasmussen, Experimental investigation of the interaction of local and overall buckling of stainless steel I-columns, ASCE J. Struct. Eng. 135 (11) (2009) 1340-1348.

[12] M. A. Wadee, L. Gardner, Cellular buckling from mode interaction in I-beams under uniform bending, Proc. R. Soc. A 468 (2137) (2012) 245-268.

[13] M. A. Wadee, L. Bai, Cellular buckling in I-section struts, Thin-Walled Struct. 81 (2014) 89-100.

[14] M. A. Wadee, M. Farsi, Cellular buckling in stiffened plates, Proc. R. Soc. A 470 (2168) (2014) 20140094. 
[15] J. M. T. Thompson, G. M. Lewis, On the optimum design of thin-walled compression members, J. Mech. Phys. Solids 20 (2) (1972) 101-109.

[16] J. M. T. Thompson, W. J. Supple, Erosion of optimum designs by compound branching phenomena, J. Mech. Phys. Solids 21 (3) (1973) 135-144.

[17] V. Tvergaard, Imperfection-sensitivity of a wide integrally stiffened panel under compression, Int. J. Solids Struct. 9 (1) (1973) 177-192.

[18] A. van der Neut, The sensitivity of thin-walled compression members to column axis imperfection, Int. J. Solids Struct. 9 (8) (1973) 999-1011.

[19] J. M. T. Thompson, G. W. Hunt, Dangers of structural optimization, Eng. Optimiz. 1 (2) (1974) 99-110.

[20] E. Byskov, J. W. Hutchinson, Mode interaction in axially stiffened cylindrical shells, AIAA J. 15 (7) (1977) 941-948.

[21] J. Loughlan, The ultimate load sensitivity of lipped channel columns to column axis imperfection, Thin-Walled Struct. 1 (1) (1983) 75-96.

[22] Z. Kołakowski, Some thoughts on mode interaction in thin-walled columns under uniform compression, Thin-Walled Struct. 7 (1) (1989) 23-35.

[23] Z. Kołakowski, Interactive buckling of thin-walled beam-columns with open and closed cross-sections, Thin-Walled Struct. 15 (3) (1993) 159-183.

[24] A. Teter, Static and dynamic interactive buckling of isotropic thin-walled closed columns with variable thickness, Thin-Walled Struct. 45 (10-11) (2007) 936-940.

[25] L. Bai, M. A. Wadee, Imperfection sensitivity of thin-walled I-section struts susceptible to cellular buckling, Int. J. Mech. Sci. 104 (2015) 162-173.

[26] M. A. Wadee, M. Farsi, Imperfection sensitivity and geometric effects in stiffened plates susceptible to cellular buckling, Structures 3 (2015) 172-186.

[27] L. Bai, M. A. Wadee, Slenderness effects in thin-walled I-section struts susceptible to local-global mode interaction, Eng. Struct. 124 (2016) 128-141.

[28] E. L. Liu, M. A. Wadee, Mode interaction in perfect and imperfect thin-walled Isection struts susceptible to global buckling about the strong axis, Thin-Walled Struct. 106 (2016) 228-243.

[29] W. T. Koiter, M. Pignataro, An alternative approach to the interaction between local and overall buckling in stiffened panels, in: B. Budiansky (Ed.), Buckling of Structures, International Union of Theoretical and Applied Mechanics, Springer Berlin Heidelberg, 1976, pp. 133-148. 
[30] E. Byskov, An asymptotic expansion applied to van der neut's column, in: J. M. T. Thompson, G. W. Hunt (Eds.), Collapse: the buckling of structures in theory and practice, Cambridge University Press, 1983, pp. 269-281.

[31] E. Byskov, Applicability of an asymptotic expansion for elastic buckling problems with mode interaction, AIAA J. 17 (6) (1979) 630-633.

[32] E. Byskov, Elastic buckling with infinitely many local modes, Mech. Struct. \& Mach. 15 (4) (1987) 413-435.

[33] S. Sridharan, Doubly symmetric interactive buckling of plate structures, Int. J. Solids Struct. 19 (7) (1983) 625-641.

[34] R. Benitot, S. Sridharan, Mode interaction in thin-walled structural members, J. Struct. Mech. 12 (4) (1984) 517-542.

[35] S. Sridharan, M. A. Ali, An improved interactive buckling analysis of thin-walled columns having doubly symmetric sections, Int. J. Solids Struct. 22 (4) (1986) 429443.

[36] M. A. Ali, S. Sridharan, A versatile model for interactive buckling of columns and beam-columns, Int. J. Solids Struct. 24 (5) (1988) 481-496.

[37] Y. K. Cheung, The finite strip method in the analysis of elastic plates with two opposite simply supported ends., Proc. Inst. Civil Eng. 40 (1968) 1-7.

[38] T. R. Graves Smith, S. Sridharan, A finite strip method for the post-locally-buckled analysis of plate structures, Int. J. Mech. Sci. 20 (12) (1978) 833-842.

[39] H. Møllmann, P. Goltermann, Interactive buckling in thin-walled beams: I. Theory, Int. J. Solids Struct. 25 (7) (1989) 715-728.

[40] P. Goltermann, H. Møllmann, Interactive buckling in thin-walled beams: II. Applications, Int. J. Solids Struct. 25 (7) (1989) 729-749.

[41] G. Kiymaz, FE based mode interaction analysis of thin-walled steel box columns under axial compression, Thin-Walled Struct. 43 (7) (2005) 1051-1070.

[42] E. L. Liu, M. A. Wadee, Interactively induced localization in thin-walled I-section struts buckling about the strong axis, Structures 4 (2015) 13-26.

[43] G. W. Hunt, M. A. Peletier, A. R. Champneys, P. D. Woods, M. A. Wadee, C. J. Budd, G. J. Lord, Cellular buckling in long structures, Nonlinear Dyn. 21 (1) (2000) $3-29$.

[44] E. L. Liu, M. A. Wadee, Geometric factors affecting I-section struts experiencing local and strong-axis global buckling mode interaction, Thin-Walled Struct. 109 (2016) 319-331. 
[45] L. Gardner, D. A. Nethercot, Experiments on stainless steel hollow sections - Part 2: Member behaviour of columns and beams, J. Constr. Steel. Res. 60 (9) (2004) $1319-1332$.

[46] D. Yang, G. J. Hancock, K. J. R. Rasmussen, Compression tests of cold-reduced high strength steel sections. II: Long columns, ASCE J. Struct. Eng. 130 (11) (2004) 1782-1789.

[47] B. Young, W. M. Lui, Tests of cold-formed high strength stainless steel compression members, Thin-Walled Struct. 44 (2) (2006) 224-234.

[48] L. Pavlovčič, B. Froschmeier, U. Kuhlmann, D. Beg, Slender thin-walled box columns subjected to compression and bending, J. Civ. Eng. Manag. 16 (2) (2010) 179-188.

[49] Y. B. Kwon, E. G. Seo, Prediction of the compressive strength of welded RHS columns undergoing buckling interaction, Thin-Walled Struct. 68 (2013) 141-155.

[50] H. X. Yuan, Y. Q. Wang, L. Gardner, Y. J. Shi, Local-overall interactive buckling of welded stainless steel box section compression members, Eng. Struct. 67 (2014) 62-76.

[51] J. Becque, The interaction of local and overall buckling of cold-formed stainless steel columns., Ph.D. thesis, University of Sydney (2008).

[52] J. Shen, M. A. Wadee, A. J. Sadowski, Interactive buckling in long thin-walled rectangular hollow section struts, Int. J. Non-Linear Mech. 89 (2017) 43-58.

[53] ABAQUS, Version 6.14, Dassault Systèmes, Providence RI, USA, 2014.

[54] P. S. Bulson, The stability of flat plates, Chatto and Windus, London, UK, 1970.

[55] J. Shen, M. A. Wadee, A. J. Sadowski, Numerical study of interactive buckling in thin-walled section box columns under pure compression, in: D. Camotim, P. B. Dinis, S. L. Chan, C. M. Wang, R. Goncalves, N. Silvestre, C. Basaglia, A. Landesmann, R. Bebiano (Eds.), Proceedings of the 8th International Conference on Advances in Steel Structures (ICASS 2015), 2015, paper number: 44.

[56] G. W. Hunt, M. A. Wadee, Localization and mode interaction in sandwich structures, Proc. R. Soc. A 454 (1972) (1998) 1197-1216.

[57] M. A. Wadee, S. Yiatros, M. Theofanous, Comparative studies of localized buckling in sandwich struts with different core bending models, Int. J. Non-Linear Mech. 45 (2) (2010) 111-120.

[58] M. A. Wadee, M. Farsi, Local-global mode interaction in stringer-stiffened plates, Thin-Walled Struct. 85 (2014) 419-430.

[59] L. Bai, M. A. Wadee, Mode interaction in thin-walled I-section struts with semi-rigid flange-web joints, Int. J. Non-Linear Mech. 69 (2015) 71-83. 
[60] G. W. Hunt, L. S. Da Silva, G. M. E. Manzocchi, Interactive buckling in sandwich structures, Proc. R. Soc. A 417 (1852) (1988) 155-177.

[61] S. P. Timoshenko, S. Woinowsky-Krieger, Theory of plates and shells, McGraw-Hill, New York, 1959.

[62] E. J. Doedel, B. E. Oldeman, AUTO-07p: Continuation and bifurcation software for ordinary differential equations, available from http://indy.cs.concordia.ca/auto/ (2009).

[63] S. Yiatros, O. Marangos, M. A. Wadee, C. Georgiou, Localized buckling in sandwich struts with inhomogeneous deformations in both face plates, Compos. Struct. 133 (2015) 630-641.

[64] E. Riks, An incremental approach to the solution of snapping and buckling problems, Int. J. Solids Struct. 15 (7) (1979) 529-551.

[65] L. G. Brazier, On the flexure of thin cylindrical shells and other "thin" sections, Proc. R. Soc. A 116 (773) (1927) 104-114.

[66] M. K. Wadee, M. A. Wadee, A. P. Bassom, A. A. Aigner, Longitudinally inhomogeneous deformation patterns in isotropic tubes under pure bending, Proc. R. Soc. A 462 (2067) (2006) 817-838.

[67] G. Garcea, L. Leonetti, D. Magisano, R. Gonalves, D. Camotim, Deformation modes for the post-critical analysis of thin-walled compressed members by a Koiter semianalytic approach, Int. J. Solids Struct. 110-111 (2017) 367-384. 